\title{
Genomic characterization and phylogenetic analysis of Salmonella enterica serovar Javiana
}

\author{
Lauren K Hudson $^{1}$, Lisha Constantine-Renna ${ }^{2}$, Linda Thomas ${ }^{3}$, Christina Moore $^{3}$, Xiaorong Qian ${ }^{3}$, Katie Garman $^{2}$ \\ , John R Dunn ${ }^{2}$, Thomas G Denes ${ }^{\text {Corresp. } 1}$ \\ 1 Department of Food Science, University of Tennessee, Knoxville, Tennessee, United States of America \\ 2 Tennessee Department of Health, Nashville, Tennessee, United States of America \\ 3 Division of Laboratory Services, Tennessee Department of Health, Nashville, Tennessee, United States of America
}

Corresponding Author: Thomas G Denes

Email address: tdenes@utk.edu

Salmonella enterica serovar Javiana is the fourth most reported serotype of laboratoryconfirmed human Salmonella infections in the U.S. and in Tennessee (TN). Although Salmonella ser. Javiana is a common cause of human infection, the majority of cases are sporadic in nature rather than outbreak-associated. To better understand Salmonella ser. Javiana microbial population structure in TN, we completed a phylogenetic analysis of 111 Salmonella ser. Javiana clinical isolates from TN collected from Jan. 2017 to Oct. 2018. We identified mobile genetic elements and genes known to confer antibiotic resistance present in the isolates, and performed a pan-genome-wide association study (pan-GWAS) to compare gene content between clades identified in this study. The population structure of TN Salmonella ser. Javiana clinical isolates consisted of three genetic clades: TN clade I $(n=54)$, TN clade II $(n=4)$, and TN clade III $(n=48)$. Using a 5,10 , and 25 hqSNP distance threshold for cluster identification, nine, 12, and 10 potential epidemiologically-relevant clusters were identified, respectively. The majority of genes that were found to be overrepresented in specific clades were located in mobile genetic element (MGE) regions, including genes encoding integrases and phage structures (91.5\%). Additionally, a large portion of the over-represented genes from TN clade II (44.9\%) were located on an $87.5 \mathrm{~kb}$ plasmid containing genes encoding a toxin/antitoxin system ( $c c d A B)$. Additionally, we completed phylogenetic analyses of global Salmonella ser. Javiana datasets to gain a broader insight into the population structure of this serovar. We found that the global phylogeny consisted of three major clades (one of which all of the TN isolates belonged to) and two cgMLST eBurstGroups (ceBGs) and that the branch length between the two Salmonella ser. Javiana ceBGs (1,423 allelic differences) was comparable to those from other serovars that have been reported as polyphyletic (929-2,850 allelic differences). This study demonstrates the population structure of TN and global Salmonella ser. Javiana 
isolates, a clinically important Salmonella serotype, and can provide guidance for phylogenetic cluster analyses for public health surveillance and response. 
1

2 Genomic Characterization and Phylogenetic Analysis

3 of Salmonella enterica Serovar Javiana

4

5

6 Lauren K. Hudson ${ }^{1}$, Lisha Constantine-Renna ${ }^{2}$, Linda Thomas ${ }^{3}$, Christina Moore ${ }^{3}$, Xiaorong

7 Qian $^{3}$, Katie Garman ${ }^{2}$, John R. Dunn ${ }^{2}$, and Thomas G. Denes ${ }^{1}$

8

$9 \quad{ }^{1}$ Department of Food Science, University of Tennessee, Knoxville, TN, USA

$10{ }^{2}$ Tennessee Department of Health, Nashville, TN, USA

$11{ }^{3}$ Tennessee Department of Health, Division of Laboratory Services, Nashville, TN, USA

12

13 Corresponding Author:

14 Thomas Denes

152600 River Drive, Knoxville, TN, 37996-4591, USA

16 Email address: tdenes@utk.edu 


\section{Abstract}

18

19

20

21

22

23

24

25

26

27

28

29

30

31

32

33

34

35

36

37

38

39

40

41

42

43

44

45

46

47

48

49

50

51

52

53

54

55

56

Salmonella enterica serovar Javiana is the fourth most reported serotype of laboratoryconfirmed human Salmonella infections in the U.S. and in Tennessee (TN). Although Salmonella ser. Javiana is a common cause of human infection, the majority of cases are sporadic in nature rather than outbreak-associated. To better understand Salmonella ser. Javiana microbial population structure in TN, we completed a phylogenetic analysis of 111 Salmonella ser. Javiana clinical isolates from TN collected from Jan. 2017 to Oct. 2018. We identified mobile genetic elements and genes known to confer antibiotic resistance present in the isolates, and performed a pan-genome-wide association study (pan-GWAS) to compare gene content between clades identified in this study. The population structure of TN Salmonella ser. Javiana clinical isolates consisted of three genetic clades: TN clade I $(n=54)$, TN clade II $(n=4)$, and TN clade III $(n=48)$. Using a 5, 10, and 25 hqSNP distance threshold for cluster identification, nine, 12, and 10 potential epidemiologically-relevant clusters were identified, respectively. The majority of genes that were found to be over-represented in specific clades were located in mobile genetic element (MGE) regions, including genes encoding integrases and phage structures (91.5\%). Additionally, a large portion of the over-represented genes from TN clade II (44.9\%) were located on an 87.5 $\mathrm{kb}$ plasmid containing genes encoding a toxin/antitoxin system $(c c d A B)$. Additionally, we completed phylogenetic analyses of global Salmonella ser. Javiana datasets to gain a broader insight into the population structure of this serovar. We found that the global phylogeny consisted of three major clades (one of which all of the TN isolates belonged to) and two cgMLST eBurstGroups (ceBGs) and that the branch length between the two Salmonella ser. Javiana ceBGs (1,423 allelic differences) was comparable to those from other serovars that have been reported as polyphyletic (929-2,850 allelic differences). This study demonstrates the population structure of TN and global Salmonella ser. Javiana isolates, a clinically important Salmonella serotype, and can provide guidance for phylogenetic cluster analyses for public health surveillance and response.

\section{Introduction}

Salmonella enterica subspecies enterica serovar Javiana (Salmonella ser. Javiana) was ranked the fourth most reported serotype (behind Enteritidis, Typhimurium, and Newport) in the United States in 2015, accounting for 7.4\% $(\mathrm{n}=575)$ of laboratory confirmed human Salmonella infections (Centers for Disease Control and Prevention (CDC), 2017a). The incidence rate (IR) was 1.17 per 100,000 persons (Centers for Disease Control and Prevention (CDC), 2017a). In 2016, 2,719 culture-confirmed human Salmonella ser. Javiana infections were reported to the Laboratory-based Enteric Disease Surveillance (LEDS) system (9.8\% of Salmonella infections; IR of 1.43 per 100,000 persons) (Centers for Disease Control and Prevention (CDC), 2018). The number of actual illnesses is likely higher according to CDC estimates of 29.3 actual cases per each laboratory-reported case (Scallan et al., 2011). Nationally, Salmonella ser. Javiana IR 
57 increased 136\% from 2001 to 2016 and 325\% since 1970 (Centers for Disease Control and 58 Prevention (CDC), 2018). From a total of 2,390 Salmonella ser. Javiana cases in FoodNet states 59 that occurred from 1996 to 2006, 20.6\% resulted in hospitalization, 2.8\% in invasive disease, and $60 \quad 0.4 \%$ in death (Jones et al., 2008). Infections in infants and young children occur at higher rates 61 than other Salmonella serotypes (Jones et al., 2008; Shaw et al., 2016; Srikantiah et al., 2004).

62 Javiana is also the fourth most common clinically isolated Salmonella serovar in Tennessee 63 (TN), accounting for $7.8 \%$ of culture-confirmed Salmonella infections in 2016 (Centers for 64 Disease Control and Prevention (CDC), 2018). The IR for TN reported by LEDS in 2016 was 651.17 (per 100,000 population), with a total of 74 culture-confirmed human infections reported 66 (Centers for Disease Control and Prevention (CDC), 2018).

67

68

69

70

71

72

73

74

75

76

77

78

79

80

81

82

83

84

85

86

87

88

89

90

91

92

93

94

95

96

Salmonella ser. Javiana is commonly found in the South Eastern US (Centers for Disease Control and Prevention (CDC), 2013; Reddy et al., 2016), particularly South Georgia (GA), Central Arkansas, and coastal areas of South and North Carolina (Srikantiah et al., 2004). States with the highest IR (per 100,000 population) of culture-confirmed human Salmonella ser. Javiana reported to LEDS in 2016 were Mississippi (6.65), South Dakota (6.29), South Carolina (5.17), North Carolina (3.50), and Georgia (3.50) (Centers for Disease Control and Prevention (CDC), 2018). Geographical restriction or distribution, as seen for Salmonella ser. Javiana, may indicate association with local food products, persistence in or adaptation to specific regional environments, and/or an environmental or animal reservoir with a specific geographic territory (Clarkson et al., 2010; Reddy et al., 2016; Strawn et al., 2014). Salmonella ser. Javiana cases are typically highest in summer (Clarkson et al., 2010; Reddy et al., 2016; Srikantiah et al., 2004), suggesting higher populations of an animal reservoir, higher exposure to sources or reservoirs, and/or increased contamination levels (Boore et al., 2015).

Due to the geographical distribution of this serovar, some researchers have evaluated correlations between incidence and environmental conditions or potential contamination sources. Huang, et al. described the association between wetlands and incidence of Salmonella ser. Javiana (Huang et al., 2017). They found that freshwater forested/scrub-shrub wetland and freshwater emergent wetland were both significantly associated with increased Salmonella ser. Javiana IR in GA, Maryland (MD), and TN. Additionally, freshwater pond wetlands were both significantly associated with increased IR in GA and TN. Shaw, et al. found a statistically significant association between increased rates of Salmonella ser. Javiana infection and percentage of housing in rural areas in Georgia (Shaw et al., 2016). Furthermore, they found a statistically significant association between increased Salmonella ser. Javiana infection rates and the presence of broiler feeding operations in MD (Shaw et al., 2016). Rural areas in GA and MD have a high density of broiler chicken operations and rural areas in TN have a high density of both broiler chicken and cattle operations (Shaw et al., 2016). The higher presence of these operations in rural areas could facilitate transmission of Salmonella ser. Javiana and other serovars in a variety of ways. As these operations are found in high density in these rural areas, 
97 they may employ a large number of residents in the area. These employees may be directly 98 exposed to Salmonella occupationally and indirectly expose others in those communities via 99 items like clothes and shoes (Shaw et al., 2016). The high density of these operations could also 100 lead to environmental transmission via contamination of groundwater and surface water with

101

102

103

104

105

106

107

108

109

110

111

112

113

114

115

116

117

118

119

120

121

122

123

124

125

126

127

128

129

130

131

132

133

134

135

136 untreated animal waste (Shaw et al., 2016). Shaw, et al. did not find any statistically significant correlations between rurality or presence of broiler, cattle, dairy, or hog operations and IR ratios of Salmonella ser. Javiana in TN (Shaw et al., 2016).

Salmonella ser. Javiana outbreaks have been linked to chicken (Jackson et al., 2013), pork (Jackson et al., 2013), cheese (Alley \& Pijoan, 1942; Hedberg et al., 1992), shrimp (Venkat et al., 2018), produce (Bennett et al., 2015; Blostein, 1993; Centers for Disease Control and Prevention (CDC), 2005; Centers for Disease Control and Prevention (CDC), 2007; Hanning et al., 2009; Jackson et al., 2013; Sandt et al., 2006; Sivapalasingam et al., 2004; Srikantiah et al., 2005; Toth et al., 2002; Walsh et al., 2014), spices (Lehmacher et al., 1995; Zweifel \& Stephan, 2012), ill foodhandlers (Elward et al., 2006; Lee et al., 1998), and contact with amphibians (Srikantiah et al., 2004). According to the National Outbreak Reporting System (NORS), there have been eight Salmonella ser. Javiana outbreaks involving TN, five multistate and three singlestate and all were foodborne. Identified vehicles included tomatoes, cucumbers, tilapia, fajita (beef), and iceberg lettuce. All of the TN outbreaks were in restaurant settings.

Salmonella ser. Javiana has been isolated from a variety of foods, including seafood (Mezal et al., 2013), white pepper (Mezal et al., 2013), produce (Duffy et al., 2005; Elviss et al., 2009; Mezal et al., 2013; Reddy et al., 2016), and pecans (Brar et al., 2016). Environmentally, Salmonella ser. Javiana has been isolated from surface water and sediment (Bell et al., 2015; Li et al., 2014; Micallef et al., 2012), poultry farms (Gama et al., 2003; Rodriguez et al., 2006; Santos et al., 2007), dairy and livestock farms (Adesiyun et al., 1996; Oliveira et al., 2002; Rodriguez et al., 2006), irrigation water (Duffy et al., 2005), and packing shed equipment surfaces (Duffy et al., 2005). It has also been recovered from wildlife (Drake et al., 2013; Gruszynski et al., 2014; Lockhart et al., 2008; Miller et al., 2014), pets (Adesiyun et al., 1997; Leahy et al., 2016; Seepersadsingh et al., 2004; Woodward et al., 1997), and zoo animals (Gopee et al., 2000). The diversity of animals found carrying Salmonella ser. Javiana includes amphibians (Drake et al., 2013), reptiles (Lockhart et al., 2008; Woodward et al., 1997), birds (Gopee et al., 2000; Gruszynski et al., 2014), and mammals (Adesiyun et al., 1997; Gopee et al., 2000; Gruszynski et al., 2014; Iveson \& Bradshaw, 1973; Leahy et al., 2016; Miller et al., 2014; Seepersadsingh et al., 2004). As Salmonella ser. Javiana has been isolated from and associated with contact with reptiles and amphibians, this may play a role in contamination of plant-based food commodities (Centers for Disease Control and Prevention (CDC), 2002; Clarkson et al., 2010; Jackson et al., 2013). A recently published systematic review identified the following risk factors associated with Salmonella ser. Javiana infection: consumption of fresh produce (tomatoes and watermelons), herbs (paprika-spice), dairy products (cheese), drinking 
137 contaminated well water, and animal contact (Mukherjee et al., 2019). Clarkson, et al. found

138

139

140

141

142

143

144

145

146

147

148

149

150

151

152

153

154

155

156

157

158

159

160

161

162

163

164

165

166

167

168

169

170

171

172

173

174

175

176 consumption of well water, reptile/amphibian contact, and exposure to recreational water associated with Salmonella ser. Javiana infection in GA and TN, but found consumption of tomatoes and poultry protective (Clarkson et al., 2010).

Though antibiotics are generally not used to treat uncomplicated Salmonella infections, when necessary, antibiotics most commonly used include ampicillin (penicillin), chloramphenicol (phenicol), ciprofloxacin (fluoroquinolone), ceftriaxone (cephalosporin), trimethoprim-sulfamethoxazole (folate pathway inhibitor, sulfonamide), amoxicillin (penicillin), and azithromycin (macrolide) (Cuypers et al., 2018; Eng et al., 2015; Gilbert et al., 2016; Jajere, 2019; Shane et al., 2017). The 2019 "Antibiotic Resistance Threats in the United States" report lists drug-resistant nontyphoidal Salmonella as a "serious threat" and details increasing numbers of isolates ciprofloxacin nonsusceptible, ceftriaxone resistant, or with decreased susceptibility to azithromycin (Centers for Disease Control and Prevention (CDC), 2019a). From the National Antimicrobial Resistance Monitoring System (NARMS) Now Salmonella ser. Javiana human isolate data from 1996-2019 (Centers for Disease Control and Prevention (CDC), 2019b), the highest prevalences of phenotypic antibiotic resistance were to streptomycin $(2.27 \%$; aminoglycoside), ampicillin (1.39\%), and tetracycline (1.05\%). Resistance to amoxicillinclavulanic acid, cefoxitin (cephalosporin), ceftiofur (cephalosporin), ceftriaxone, cephalothin (cephalosporin), chloramphenicol, sulfamethoxazole/sulfisoxazole (sulfonamides), and trimethoprim-sulfamethoxazole were all less than 1\% (Centers for Disease Control and Prevention (CDC), 2019b). Resistance to azithromycin or ciprofloxacin was not reported (Centers for Disease Control and Prevention (CDC), 2019b). Resistance among Salmonella ser. Javiana isolates may be lower due to the association with wild animal (e.g., reptile and amphibian) and other environmental reservoirs in contrast to food animal-associated serovars.

Though Salmonella ser. Javiana is a prevalent serotype in both the US and TN, little is known about the genomic population structure. The objectives of this study were to retrospectively study isolates of Salmonella ser. Javiana from patients in TN in 2017-2018 in order to identify epidemiologically-relevant trends, determine the genomic population structure, and describe the defining genomic features of major clades. Additionally, we studied expanded datasets representing global diversity to determine the overall population structure of Salmonella ser. Javiana and to compare it to other Salmonella serovars.

\section{Materials \& Methods}

Sequencing, preprocessing, and genome assembly of TN isolates. BioSample numbers and metadata for Salmonella ser. Javiana $(n=111)$ isolates from patients in TN from January 2017 through October 2018 were obtained from the Tennessee Department of Health (TDH) (Data S1). Tennessee population data (2018) used for calculating incidence rates (IR) was 
177 obtained from the U.S. Census Bureau (U.S. Census Bureau) and IR per county were mapped 178 using Tableau Desktop Public Edition (v2019.2.1) (Tableau Software, 2019). PFGE and whole179 genome sequencing were performed by the TDH Division of Laboratory Services according to 180 PulseNet protocols (Centers for Disease Control and Prevention (CDC), 2016; Centers for 181 Disease Control and Prevention (CDC), 2017b). For PFGE, XbaI was used as the primary 182 restriction enzyme. Genomic DNA was extracted using Qiagen DNeasy Blood \& Tissue kits, 183 libraries were prepared using Nextera XT kits, and sequencing was performed on an Illumina 184 MiSeq platform using Illumina MiSeq v2 chemistry (500 cycle) to produce $250 \mathrm{bp}$ paired-end 185 reads. Raw reads were downloaded from the NCBI SRA database, trimmed using Trimmomatic v0.35 (Bolger et al., 2014) (with the following parameters: ILLUMINACLIP: NexteraPEPE.fa:2:30:10 LEADING:3 TRAILING:3 SLIDINGWINDOW:4:15 MINLEN:36), and quality checked using FastQC v0.11.7 (Andrews, 2010) and MultiQC v1.5 (Ewels et al., 2016) to combine the results. The trimmed reads were assembled into contigs using SPAdes v3.12.0 (Bankevich et al., 2012) with the careful option. Assembly statistics were generated by BBMap v38.88 (Bushnell, 2018), SAMtools v0.1.8 (Li et al., 2009), and QUAST v4.6.3 (Gurevich et al., 2013). SeqSero (Zhang et al., 2015) was used to confirm serotype designations.

196

197

198

199

200

201

202

203

204

205

206

207

208

209

210

211

212

213

214

215

216

SNP Detection and phylogenetic analyses. A reference-free SNP detection analysis was initially performed with the TN isolates to determine the overall population structure free of reference choice bias. The assemblies were analyzed using KSNP3.1 (Gardner et al., 2015) and the resulting core SNP matrix fasta file was then used to construct a phylogenetic tree in Mega7 (Kumar et al., 2016) with 100 bootstrap replicates (Felsenstein, 1985). The evolutionary distances were computed using the number of differences method (Nei \& Kumar, 2000) and the evolutionary history was inferred using the Neighbor-Joining method (Saitou \& Nei, 1987). The final tree was visualized and annotated using iTOL (Letunic \& Bork, 2016). Isolates that weren't serotype Javiana (based on SeqSero results) and were very divergent based on the KSNP analysis were removed from the analysis. Major clades (defined as groups of three or more isolates that were all within 500 SNPs of each other) were identified. Next, reference-based hqSNP analyses were performed for each TN clade independently to determine high-resolution SNP differences between isolates. For the hqSNP analyses, an appropriate internal reference genome assembly (with adequate assembly quality and expected assembly size and $\mathrm{G}+\mathrm{C}$ content) for each clade was identified (SRS2420927 for TN clade I, SRS2822480 for TN clade II, and SRS3010019 for TN clade III). Additionally, the Salmonella enterica subsp. enterica serovar Javiana str. CFSAN001992 assembly (GCF_000341425.1) was downloaded from the NCBI RefSeq database for use as an external and closed reference genome. The hqSNP analyses were performed, both with the internal and external references and for the 111 isolates together and for each TN clade individually. For each analysis, high quality single nucleotide polymorphisms (hqSNPs) were identified using the CFSAN SNP Pipeline v1.0.1 (Davis et al., 2015). The resulting hqSNP matrix fasta files were then used to construct phylogenetic trees as described above. The matrices were sorted and clustered using the hclust function (gtools package) in R studio. For the 
217 individual clade analyses using internal references, clusters of two or more related isolates were

218

219

220

221

222

223

224

225

226

227

228

229

230

231

232

233

234

235

236

237

238

239

240

241

242

243

244

245

246

247

248

249

250

251

252

253

254

255

256

identified at hqSNP distance threshold levels of 5, 10 and 25; isolation date and other epidemiological information were not considered.

Genome annotation and pan-GWAS. TN isolate assemblies were annotated using Prokka v1.14-dev (Seemann, 2014) and RASTtk (Brettin et al., 2015). A pangenome-wide association study (pan-GWAS) was performed to compare gene content among the isolates using Roary v3.12.0 (with Prokka annotation output files, previously described, used as input files) (Page et al., 2015) and statistical analysis was done using Scoary v1.6.16 (with the following arguments: -c I B BH PW EPW P -p 0.05 -e 100) (Brynildsrud et al., 2016) to identify genes or markers associated with inclusion in each clade. Genes predominantly present or absent among isolates in each clade were identified. Genes or clusters of genes (loci) were considered significantly associated with a clade or cluster if they had a Bonferroni-corrected P-value $<0.05$. From the pan-GWAS, the positively and negatively associated genes were classified by having an Odds Ratio of $>1$ and $<1$, respectively. To further analyze the results in a genomic context, loci that were adjacent or located in close proximity were combined into a single region.

In silico Identification of genomic features and genes. Phage regions were identified in a diverse representative subset of the TN isolates $(n=31)$ using Phaster (Arndt et al., 2016; Zhou et al., 2011). Potential plasmids were predicted and classified using PlasmidFinder v2.0.2 (database version 2019-05-16) (Carattoli et al., 2014), Unicycler v0.4.8-beta (Wick et al., 2017), and plasmidSPAdes (Antipov et al., 2016) and visualized using Bandage (Wick et al., 2015). They were further confirmed by examining the associated assembly contigs for plasmidassociated genes, comparing to known plasmids using PLSDB (Galata et al., 2018) and BLASTn, and comparing coverage and $\mathrm{G}+\mathrm{C}$ content to the whole assembly. Antibiotic resistance (ABR) determinants in genomes were predicted using ResFinder ( $90 \%$ threshold for identity and $60 \%$ for minimum length) (Zankari et al., 2012) to identify acquired ABR genes and PointFinder (90\% threshold for identity and 60\% for minimum length) (Zankari et al., 2017) to identify point mutations conferring ABR. The representative subset of the isolates $(n=31)$ was also examined for virulence factors using VirulenceFinder (Joensen et al., 2014) and VFDB VFanalyzer (Liu et al., 2018) and Salmonella Pathogenicity Islands (SPIs) using SPIFinder (v1.0; 95\% threshold for identity and 60\% for minimum length) (Roer et al., 2016).

Global phylogenetic analysis. The Salmonella database (Alikhan et al., 2018) on EnteroBase (Zhou et al., 2019) was queried for isolates with "human" listed as source niche in the strain metadata and "Javiana" listed as serovar in the strain metadata or experimental data (SISTR1 (Yoshida et al., 2016) or SeqSero2 (Zhang et al., 2019a)). Only strains with country (and state for strains from the United States) included in the metadata were retained. A cgMLST + HierCC minimal spanning tree (RapidNJ algorithm) was created with GrapeTree (Zhou et al., 2018) on EnteroBase using all of the resulting strains. Strains that were likely not Javiana

Peer] reviewing PDF | (2020:05:49215:2:0:NEW 30 Sep 2020) 
257 (conflicting serovar designations and distant on the tree) were removed from the dataset, leaving 258466 strains (Data S2). The dataset was further refined to select representative strain(s) for each 259 HC100 level cluster ( $\leq 100$ cgMLST allelic differences). For each HC100 cluster, a single strain 260 from each country/state was retained. If there were more than one strain from a country/state, the

261

262

263

264

265

266

267

268

269

270

271

272

273

274

275

276

277

278

279

280

281

282

283

284

285

286

287

288

289

290

291

292

293

294

295

296 representative strain was chosen based on assembly quality (N50; and if N50 values were identical or similar, coverage and number of contigs were also considered). At least one TN strain representing each $\mathrm{HC} 100$ cluster (if available) was chosen to be included in the final dataset of genomes representing global diversity of of Salmonella ser. Javiana clinical isolates. The final dataset consisted of 162 strains: 29 TN isolates ( 11 from TN clade I, 3 from TN clade II, 10 from TN clade III, and the five isolates that didn't fall into the main clades), 43 strains from other states in the US, and 90 isolates from other countries (Data S2). Collection date years ranged from 2002 to 2020. Assemblies for the non-TN strains were downloaded from Enterobase. All assemblies were analyzed using KSNP3.1 (Gardner et al., 2015) and the resulting core SNP matrix fasta file was then used to construct a phylogenetic tree in Mega7 (Kumar et al., 2016) with 100 bootstrap replicates (Felsenstein, 1985). The evolutionary distances were computed using the number of differences method (Nei \& Kumar, 2000) and the evolutionary history was inferred using the Neighbor-Joining method (Saitou \& Nei, 1987). The final tree was visualized and annotated using iTOL (Letunic \& Bork, 2016).

Comparison to polyphyletic serovars. Eight strain datasets were created: one for serovar Javiana and one each for other serovars that have been reported as polyphyletic (Derby, Kentucky, Mississippi, Montevideo, Newport, Saintpaul, and Senftenberg). The Salmonella database (Alikhan et al., 2018) on EnteroBase (Zhou et al., 2019) was queried for isolates with the specified serovar listed as serovar in the experimental data (SISTR1 (Yoshida et al., 2016) or SeqSero2 (Zhang et al., 2019a)). For each, a cgMLST + HierCC minimal spanning tree (RapidNJ algorithm) was created. Strains that were likely not the serovar of interest (conflicting serovar designations and distant on the tree) were removed from the datasets. The final datasets (Data S3) were used to create cgMLST + HierCC minimal spanning trees (improved minimal spanning tree algorithm, MSTree V2) using GrapeTree (Zhou et al., 2018) on EnteroBase. The branch lengths between the cgMLST eBurstGroups (ceBGs) of the other polyphyletic serovars were used for comparison to the branch length between the two Javiana ceBGs. ceBG designations associated with each serovar were retrieved from the EnteroBase documentation (EnteroBase Team, 2018).

\section{Results}

Tennessee Salmonella ser. Javiana Population Structure. This analysis included a diverse set of 111 Salmonella ser. Javiana clinical isolates from TN (Data S1). On average, the assembled genomes from this study had $74.6 x$ coverage, contained 71.7 contigs $(34.8$ contigs $\geq 1$ $\mathrm{kb}$ ), were $46.45 \mathrm{~kb}$ in length, and had $52.11 \%$ GC content (Data S1). Based on the KSNP 
297

298

299

300

301

302

303

304

305

306

307

308

309

310

311

312

313

314

315

316

317

318

319

320

321

322

323

324

325

326

327

328

329

330

331

332

333

334

335

analysis, the Salmonella ser. Javiana isolates from TN displayed a population structure with three main clades (Fig. 1). TN Clade I contained 54 isolates, TN clade II contained four, TN clade III contained 48 isolates, and five isolates didn't fall into the main clades (Fig. 1 and Table 1). Isolates in TN clades I, II, and III had average hqSNP distances of 119.4 (range 0 to 631), 210.3 (range 3 to 396), and 66 (range 0 to 361), respectively (Table 1 and Data S4).

The 111 TN Salmonella ser. Javiana clinical isolates represented 47 different PFGE patterns (Fig. 2; Data S1). The most common PFGE patterns were JGGX01.0012 ( $\mathrm{n}=18)$, JGGX01.0065 $(\mathrm{n}=17)$, and JGGX01.0072 $(\mathrm{n}=10)$. TN clade I isolates represented 28 different PFGE patterns, with the most common being JGGX01.0012 $(\mathrm{n}=18)$. TN clade II isolates represented three different PFGE patterns. TN clade III isolates represented 11 different PFGE patterns, with the most common being JGGX01.0065 $(n=17)$ and JGGX01.0072 $(n=10)$. Each of the five isolates that did not belong to a clade had a distinct PFGE pattern. All PFGE patterns were unique to only one TN clade.

High-quality single nucleotide polymorphism (hqSNP) analysis for cluster detection. Using a 5, 10, and 25 hqSNP distance threshold for cluster identification, 9, 12, and 10 potential clusters were identified, respectively (Data S4). The number of clusters decreases from thresholds of 10 to 25 , as with the larger threshold, some of the clusters contain multiple subclusters identified at the lower threshold. Within TN clade I, five clusters were identified at each hqSNP distance thresholds of 5 and 10, and four clusters at 25 (Data S4). Only one cluster was identified at each of the distance thresholds for TN clade II (Data S4). Within TN clade III, three clusters were identified at a distance threshold of 5 hqSNPs, six clusters at 10 hqSNPs, and five clusters at hq25 SNPs (Data S4).

To evaluate the effects of reference choice and isolate diversity, we ran our hqSNP analyses on the entire TN dataset (111 isolates) and on the three clades individually and with both internal draft assemblies and an external closed assembly as reference genomes. When all isolates were analyzed together using different reference genomes, the average hqSNP distance was lowest when using the internal reference from TN clade I and highest when using the external closed genome and the average percentage of reads mapped differed by up to $1.57 \%$ (Table 1 and Data S4). It should be noted that the closed external reference assembly we used (GCF_000341425.1) was most closely related to TN clade II in our original KSNP analysis (Fig. 1) and not representative of the overall population (at the time that this analysis was performed, there was only one closed Salmonella ser. Javiana genome available on NCBI RefSeq). For all three clades, regardless of the reference genome used, hqSNP distances were higher when they were analyzed independently, with the differences being only slightly higher for clades I and III. The average hqSNP distances for clades I and II were lower when using the internal references, but were slightly higher for TN clade III. For all three clades, the average percent reads mapped 
336

337

338

339

340

341

342

343

344

345

346

347

348

349

350

351

352

353

354

355

356

357

358

359

360

361

362

363

364

365

366

367

368

369

370

371

372

373

374

375

was higher when using the internal reference genome than the external reference genome, which is to be expected.

Epidemiological Trends. The TN Salmonella ser. Javiana isolates were sourced from patients with an average age of 40.0 (range of 1 month to 90 years; standard deviation of 29.1). The highest incidence was in patients $\leq 4$ (6.64 per 100,000) and $\geq 85$ (4.16) years-old. Previous studies have reported that Salmonella ser. Javiana infections are more prevalent in infants and young children than for other serovars (Jones et al., 2008; Shaw et al., 2016; Srikantiah et al., 2004). Overall, $51.4 \%$ of isolates were from male patients (incidence of 1.73 ) and $46.8 \%$ from female (incidence of 1.50; Table 2). TN clades I and II contained more isolates from male patients than female, $57.4 \%$ and $75 \%$, respectively. Conversely, $52.1 \%$ of TN clade III isolates were from female patients.

Most of the TN Salmonella ser. Javiana isolates were taken from stool samples $(84.7 \%$, $\mathrm{n}=94)$, followed by urine $(6.3 \%, \mathrm{n}=7)$ and blood $(5.4 \%, \mathrm{n}=6)$ (Table 2$)$. The portion of isolates taken from blood samples exceeds the $2.8 \%$ invasive disease outcome (defined by isolation from blood, cerebrospinal fluid, bone or joint fluid, or another sterile site; does not include urine, wound, abscess cultures) reported for this serovar in FoodNet states (Jones et al., 2008). Of the urine isolates, most $(n=6)$ belonged to TN clade III and all were from females, with an average patient age of 55.1 (standard deviation of 26.2). All of the isolates recovered from blood samples belonged to TN clades I $(n=3)$ and III $(n=3)$ and most were from males $(n=4)$, with an average patient age of 71.8 (standard deviation of 15.2). The majority of isolates recovered from extraintestinal sites were collected from elderly patients, which may indicate a correlation between invasive infection and age.

Geographical and Temporal Distribution. A geographical distribution can be seen for the TN isolates, with $65.8 \%(\mathrm{n}=73)$ isolated in counties in the western region of TN (Table 2; Data S1). In contrast, only $17.1 \%(n=19)$ and $16.2 \%(n=18)$ were isolated in counties in east and middle TN, respectively. Incorporating county population data, the west region had an IR of 4.69 clinical isolates per 100,000 population, while the east had an IR of 0.79 and the middle had an IR of 0.64, with an overall IR of 1.62 per 100,000 for the state (Fig. S2). Three counties had noticeably higher IR: Madison with 31.0, Crockett with 27.9, and Carroll with 25.0. As was the trend for all isolates, the majority of TN clade I and III isolates originated in the west region (59.3\% and $83.3 \%$, respectively; Table 2$)$. However, a sizable amount of TN clade I isolates also originated in the east region (25.9\%). Additionally, a temporal distribution was also clear, with $68.5 \%(n=76)$ of isolates collected in July through September (Table 2; Data S1). This trend was also seen within the three TN clades.

Identification of Mobile Genetic Elements (MGEs). All of the TN isolates were examined for plasmids and 32 putative plasmids (19 unique) were identified in 30 isolates 
376 (Table 3). They ranged in size from 23 to $108 \mathrm{~kb}$ and included replicon types (a plasmid typing 377 scheme based on replication control regions (Carattoli et al., 2014)) IncFIB, IncFII, IncI1, IncN3, 378 and IncX4. In the representative subset of TN isolates $(n=31)$, Phaster predicted an average of 3798.90 [range of 5-13] prophage regions per isolate (2.29 intact, 4.94 incomplete, and 1.68 380 questionable) (Data S5). The TN clade I isolates had the highest number of predicted prophage 381 regions (average of 9.64 and range of 8-11), followed by TN clade II (average of 8.25 and range

382

383

384

385

386

387

388

389

390

391

392

393

394

395

396

397

398

399

400

401

402

403

404

405

406

407

408

409

410

411

412

413

414

415 of 8-9) and TN clade III (average of 8.09 and range of 5-10).

Identification of Virulence Factors and Pathogenicity Islands. All of the representative subset of $\mathrm{TN}$ isolates $(\mathrm{n}=31)$ contained pathogenicity islands C63PI, SPI-13, and SPI-14 (Data S6). Most of the TN isolates examined contained SPI-2 (except for SRS2442409 [TN clade II] and SRS2998834 [TN clade III]) and SPI-4 (except SRS3453943 [TN clade I], SRS3643364 [TN clade III], and SRS2998834 [TN clade III]).

Most identified virulence genes were present in all of the TN isolates analyzed (Data S6), including the three genes ( $c d t B$, pltA, and pltB) encoding the subunits of the cytolethal distending toxin (CDT) (Miller et al., 2018). Only the TN clade II isolates contained the pefC and pefD genes, which were on plasmid-associated contigs, that are part of the pef (plasmid-encoded fimbriae) operon and associated with fimbrial adherence (Bäumler et al., 1996). Some of the isolates (two from TN clade I and three that were not part of a clade) contained genes from the saf (Salmonella atypical fimbria) operon on putative plasmid-associated contigs (Folkesson et al., 1999). One isolate (SRS2442415, no clade) contained 11 genes associated with the yersiniabactin iron uptake system on a plasmid-associated contig (Carniel, 2001).

Identification of Antibiotic Resistance Genes. All 111 TN Salmonella ser. Javiana isolates analyzed in the present study contained the $a a c\left(6^{\prime}\right)$-Iaa gene, which has been previously reported to confer aminoglycoside resistance (Shaw et al., 1993). One isolate (SRS2783476; TN clade I) contained the $\operatorname{aph}\left(3^{\prime}\right)-I a$ and sul3 genes on a contig (contig 32) that associated with a putative plasmid. The aph( $\left.3^{\prime}\right)-I a$ gene has been found to confer resistance to aminoglycosides (Shaw et al., 1993) and sul3 gene has been shown to confer resistance to sulfonamides/sulfones through antibiotic target replacement and has been shown to be associated with resistance to sulfamethoxazole (Perreten \& Boerlin, 2003).

Additionally, one isolate (SRS2628542; no clade) contained the qnrB19 gene, which has been shown to confer resistance to fluoroquinolones through physical protection of the antibiotic target (Correia et al., 2017). The $q n r$ gene is plasmid-associated and has been linked with reduced susceptibility to ciprofloxacin (Casas et al., 2016; Crump et al., 2015; Redgrave et al., 2014). It is unclear if this gene in SRS2628542 is on a plasmid, as it is located on a small (703 bp) contig and no plasmids were predicted in this isolate. However, the gene showed $100 \%$ identity over only $72.6 \%$ of the alignment with the reference gene (accession EU432277), so it may not be functionally capable of conferring the quinolone resistance phenotype. 
416

417

418

419

420

421

422

423

424

425

426

427

428

429

430

431

432

433

434

435

436

437

438

439

440

441

442

443

444

445

446

447

448

449

450

451

452

453

454

455

Clade-Enriched Genes. A pangenome analysis of the TN isolates revealed that the core genome (genes contained in $\geq 99 \%$ of isolates) consisted of 4,022 genes and the accessory genome consisted of 3,920 genes (Table 1). The difference in gene content between the identified clades were mostly found in mobile genetic elements $(91.5 \%)$.

TN clade I isolates had a core genome of 4,106 genes and an accessory genome of 2,513 genes (Table 1). This clade has a much larger accessory genome than the other two clades identified in this study. This is likely due to the large variety of mobile genetic elements (i.e., plasmids, prophages) present in isolates from this clade, which is also reflected in the much larger number of PFGE patterns $(n=28)$ displayed by these isolates as compared to other clades ( $n=3$ for TN clade II and $n=11$ for TN clade III). The pan-GWAS revealed 153 loci (genes or groups of genes) to be significantly associated with inclusion in this clade (54 positively associated and 99 negatively associated) (Table 1 and Data S7). These loci consisted of 338 total genes (94 positively associated and 243 negatively associated) (Table 1). The positively associated loci are found in 29 distinct genomic regions and the majority of the genes overrepresented in TN clade I (73 genes) were located in eight prophage regions (Table 4).

Twelve of the overrepresented genes in TN clade I correspond to pathogenicity-related protein families (as identified by PathogenFinder): DNA damage-inducible protein I, phenolic acid decarboxylase subunit D, small toxic polypeptide LdrD, PTS system fructose-specific EIIB'BC component, PTS system mannose/fructose/sorbose family IID component, prepilin peptidase dependent protein A precursor, phage DNA binding protein, and other hypothetical proteins. Overexpression of $l d r D$, which is part of a chromosomal toxin-antitoxin gene system (Alix \& Blanc-Potard, 2009; Kawano et al., 2002), is toxic to the cell and leads to growth inhibition and rapid cell killing (Fozo et al., 2008; Kawano et al., 2002). ldrD homologs have not been found in plasmids, but may be involved in cellular response to environmental stress (Kawano et al., 2002). Prepilin peptidase dependent protein A precursor is known to be plasmidassociated (Raspoet et al., 2019; Zhang et al., 1994) and is involved in processing of the major pilus subunit (Filloux et al., 1998; Zhang et al., 1994). Other genes of interest enriched in isolates from TN clade I include those encoding autotransporter adhesin SadA, which is associated with pathogenesis, and virulence protein MsgA, which is involved with survival within macrophage (Skyberg et al., 2006). Mezal, et al. identified $m s g A$ virulence gene in 7 (out of 50) Salmonella ser. Javiana isolates, all of which were clinical (Mezal et al., 2013).

TN clade II isolates had a core genome of 4,290 genes and an accessory genome of 322 genes (Table 1). The pan-GWAS revealed 22 loci to be significantly associated with inclusion in this clade (16 positively associated and 6 negatively associated) (Table 1 and Data S7). These loci consisted of 221 total genes (207 positively associated and 14 negatively associated) (Table 1). The positively associated loci are found in 17 distinct genomic regions and many of the genes 
456 over-represented in TN clade II (93 genes) were located in the $87.5 \mathrm{~kb}$ IncFII type plasmid 457 identified in SRS2922480 (Table 4 and Fig. S3). Among the over-represented genes contained 458 on this plasmid are $c c d A B$, which are part of a toxin/antitoxin system. This system contributes to

459

460

461

462

463

464

465

466

467

468

469

470

471

472

473

474

475

476

477

478

479

480

481

482

483

484

485

486

487

488

489

490

491

492

493

494 stability of the plasmid through post-segregational killing (killing new cells that do not inherit a plasmid copy during cell division) (Van Melderen, 2001). Additionally, 72 over-represented genes were located in four predicted prophage regions and 33 in three other potential MGE regions (indicated by gene annotations, clustering of genes, and/or close proximity to predicted prophage regions; Table 4). One of the overrepresented genes, alpha-xylosidase, corresponds to a pathogenicity-related protein family (as identified by PathogenFinder). VFDB identified pefC and $p e f D$, fimbrial adherence determinants, on the plasmid (contig 13); $\operatorname{sinH}$, a nonfrimbrial adherence determinant; and $\operatorname{pip} B$, a TTSS-2 translocated effector.

TN clade III isolates had a core genome of 4,115 genes and an accessory genome of 889 genes (Table 1). The pan-GWAS revealed 155 loci to be significantly associated with inclusion in this clade (101 positively associated and 54 negatively associated) (Table 1 and Data S7). These groups contained 332 total genes ( 238 positively associated and 94 negatively associated) (Table 1). The positively associated loci are found in 29 distinct genomic regions and the majority of the genes over-represented in TN clade III were located in four predicted prophage regions (88 genes) or nine other putative MGE regions (134 genes) (Table 4). Four of the overrepresented genes correspond to pathogenicity-related protein families (as identified by PathogenFinder): arginine/lysine/ornithine decarboxylase) and other hypothetical proteins.

Global Population Structure. The KSNP analysis of the diverse set of global clinical Salmonella ser. Javiana strains revealed three major clades (Figure 3; Figure S1). Major clade I contained 107 strains, including TN isolates (from TN clades I, II, and III and the five isolates that didn't fall into the main clades). Major clade II contained 23 strains and major clade III contained 31 strains. Strains from major clades I and II belong to the $590 \mathrm{cgMLST}$ eBurstGroup (ceBG) and strains from major clade III belong to the $204 \mathrm{ceBG}$; both of these are associated with this serovar (EnteroBase Team, 2018).

To further explore if Salmonella ser. Javiana is polyphyletic, we constructed minimal spanning trees based on cgMLST allele distances of all available Salmonella ser. Javiana strains and of other Salmonella serovars previously described as polyphyletic (Derby, Kentucky, Mississippi, Montevideo, Newport, Saintpaul, and Senftenberg (Achtman et al., 2012; Alikhan et al., 2018; Banerji et al., 2020; Cao et al., 2013; Sangal et al., 2010; Sévellec et al., 2018; Tang et al., 2019; Timme et al., 2013; Vosik et al., 2018; Zhang et al., 2019b)) (Figure 4; Figure S2). The branch length between the two ecBG clusters on the ser. Javiana tree was 1,423 allelic differences. The branch lengths between ecBG clusters on the other trees ranged from 2,2802,769 allelic differences for ser. Derby, 2,452-2,756 for ser. Kentucky, 929-2,850 for ser.

Peer) reviewing PDF | (2020:05:49215:2:0:NEW 30 Sep 2020) 
495 Mississippi, 1,188-2,844 for ser. Montevideo, 957-2,636 for ser. Newport, 1,513-2,686 for ser.

496 Saintpaul, and 1,844-2,743 for ser. Senftenberg.

497

498 Discussion

499

500

501

502

503

504

505

506

507

508

509

510

511

512

513

514

515

516

517

518

519

520

521

522

523

524

525

526

527

528

529

530

531

532

533

534

The phylogenetic analysis of the diverse set of global clinical Salmonella ser. Javiana strains revealed three major clades (Figure 3; Figure S1). Major clade I contained most of the strains, including all of the TN isolates. This indicates that the population of this serovar in TN represents only a portion of the global genomic diversity. Strains from major clades I and II belong to the $590 \mathrm{cgMLST}$ eBurstGroup (ceBG) and strains from major clade III belong to the 204 ceBG. ceBGs are equivalent to eBurstGroups (eBGs; in legacy 7-gene MLST), which have been shown to correspond to serovar designations (EnteroBase Team, 2018; Zhou et al., 2019). Typically, monophyletic serovar isolates will belong to a single eBG, while polyphyletic serovar isolates will belong to multiple eBGs (Achtman et al., 2012; Alikhan et al., 2018; Banerji et al., 2020).The observation that the global clinical Salmonella ser. Javiana isolates consisted of multiple major clusters and two ceBGs suggests that this serovar may be polyphyletic. Ashton, et al. characterized serovars in lineage 3 of $S$. enterica subspecies I (which includes serovars Bredeney, Chester, Javiana, Montevideo, Oranienburg, and Poona) as polyphyletic and containing multiple eBGs (Ashton et al., 2016). The branch length between the two ecBG clusters on the ser. Javiana tree (1,423 allelic differences) was comparable to the branch lengths between ecBGs on the other serovar trees (929-2,850 allelic differences) (Figure 4 and Figure S2). Based on this comparison, Salmonella ser. Javiana may be considered a polyphyletic serovar, although this depends on the branch length cutoff that is applied. 
535

536

537

538

539

540

541

542

543

544

545

546

547

548

549

550

551

552

553

554

555

556

557

558

559

560

561

562

563

564

565

566

567

568

569

570

571

572

573

574

As WGS is becoming more commonly used for public health applications (e.g., cluster detection and outbreak investigation), it is important to understand genomic population structure of surveilled disease-causing microorganisms, specifically at the serovar level for Salmonella. Genomic distance thresholds (based on hqSNP or allelic distances) are an important factor used for identifying potential disease clusters of public health importance, but other factors are typically considered, including isolation date, number of isolates, and epidemiological data. In the present study, we found that using different hqSNP distance thresholds for cluster identification resulted in different numbers of potential clusters and associated isolates (Data S4). The selected threshold for cluster detection should be empirically determined so that it is larger than typical inter-genomic distances between outbreak strains, but smaller than typical inter-genomic distances between outbreak and background (non-outbreak) isolates. Intergenomic SNP distances among Salmonella outbreak strains are typically small (in the 2 to 12 SNP range), but in some cases can be quite large (up to 249 SNPs) and likely vary from serovar to serovar (Leekitcharoenphon et al., 2014). Isolates from zoonotic or prolonged (e.g., persistent contamination from production environments) outbreaks will likely have larger genomic distances and outbreaks with very large genomic distances are typically polyclonal events (Besser et al., 2019). For Salmonella, the CDC uses a working definition of $\geq 3$ cases within a 60 day period with $\leq 10$ cgMLST allele differences, with $\sim 2$ cases that have $\leq 5$ allele differences (Besser et al., 2019). Thresholds can have impacts on epidemiological investigations; if they are set too low, isolates belonging to the same outbreak event may be mistakenly excluded from the cluster or separated into different clusters and, if they are set too high, background isolates may be inadvertently included in the cluster, making epidemiological investigations difficult, particularly source attribution. In the present study, as the hqSNP distance threshold was increased, the number of included isolates also increased. Increases in numbers and/or sizes of potential clusters may impact the ability of public health departments to further investigate them due to resource constraints. Thresholds may also need to be adjusted based on the timeline of the suspected outbreak (lower for short-term and higher for prolonged outbreaks). As we move forward with using WGS for routine surveillance and cluster detection of this serovar, more clusters may be successfully detected and investigated. In turn, this will provide information on typical genomic distances that can be used to establish and evaluate an appropriate serovarspecific threshold for cluster detection.

Another important consideration when using hqSNP calling analyses for epidemiological cluster detection is whether polyphyletic serovars or those with genetically diverse clades should be analyzed together or if each clade should be analyzed independently. An additional consideration is the choice of reference genome. These choices can affect the percentage of reads mapped to the reference genome and, in turn, the results of the analysis (primarily, hqSNP distances). Better performance (i.e., higher read mapping) would be expected when using closed genomes as references for hqSNP calling. However, some research has shown that using closed vs draft genomes as references have limited impact on hqSNP calling phylogeny reconstruction

Peer] reviewing PDF | (2020:05:49215:2:0:NEW 30 Sep 2020) 
575 (Jagadeesan et al., 2019; Portmann et al., 2018). In the current study, we still achieved a level of 576 high-quality mapping ( $>95 \%$, as recommended by (Katz et al., 2017); Table 1) when using draft 577 genomes as references. As these types of studies are performed, representatives from each clade 578 should be selected for long-read sequencing to establish high quality reference genomes that can 579 be used to further evaluate hqSNP distances. Additionally, when analyzing the TN isolates 580 together or each clade separately and with internal or external reference genomes, similar levels 581 of performance were achieved. This is likely due to the lack of diversity in the core genome of 582 the isolates and the fact that the majority of the gene content differences between isolates from

583

584

585

586

587

588

589

590

591

592

593

594

595

596

597

598

599

600

601

602

603

604

605

606

607

608

609

610

611

612

613

614 each clade were attributed to MGEs (plasmids and prophage regions). Commonly used hqSNP pipelines filter out SNPs that are found in close proximity and/or mask phage regions (Katz et al., 2017; Strain et al.) and only SNPs present in genomic regions shared between isolates and the reference genome are identified in the analysis. When viewed from a global context, the TN isolates were all part of a single major clade and a single ceBG, which may also explain the similar levels of performance seen with the different analysis strategies.

Notable geographical and temporal patterns were observed for the Salmonella ser. Javiana isolates from TN. The geographical distribution within the state (most isolates from patients in counties in the western region; Table 2 and Figure S3) is consistent with other reported data (Centers for Disease Control and Prevention (CDC), 2013; Mukherjee et al., 2020). This geographical distribution may be associated with the higher percentage of fresh forested/scrub-shrub wetlands in these west TN counties (Huang et al., 2017). A similar geographical distribution has been described in GA, with Salmonella ser. Javiana cases occurring more frequently in the southern part of state (Clarkson et al., 2010). Despite this, Harris, et al. were unable to isolate Salmonella ser. Javiana from storm runoff or irrigation ponds used by fresh produce growers in South Georgia even though this is a high incidence area (Harris et al., 2018). The temporal distribution (most isolates collected July-September; Table 2) is in accordance with the notable seasonality of this serovar reported elsewhere (Clarkson et al., 2010; Srikantiah et al., 2004).

Salmonella virulence factors aid in host colonization and pathogenicity by assisting the pathogen in attaching to, invading, and replicating within host cells, intra-and extracellular survival, evading host defenses, and outcompeting the gut microbiome and include adhesion systems, capsule, flagella, and toxins (Jajere, 2019). Virulence factors and related genes are frequently clustered together in pathogenicity islands, which are often found on mobile genetic elements (MGEs), such as plasmids and prophages (Cheng et al., 2019; Jacobsen et al., 2011). Eight Salmonella Pathogenicity Islands (SPIs) or islets (SPI-1, SPI-2, SPI-4, SPI-5, SPI-9, SPI11, SPI-12 and CS54) are commonly found in most non-typhoidal serovars (den Bakker et al., 2011; Jacobsen et al., 2011). All of the representative subset of TN isolates analyzed for SPIs contained C63PI, SPI-13, and SPI-14 (Data S6). C63PI, which is located within SPI-1, contains the sit operon that encodes an iron uptake system (Schmidt \& Hensel, 2004; Zhou et al., 1999). 
615 SPI-13 has been associated with macrophage internalization and virulence in chickens and mice 616 (Cheng et al., 2019; Elder et al., 2016; Espinoza et al., 2017; Shah et al., 2005). SPI-14 is 617 involved in epithelial invasion and pathogenicity in chickens (Cheng et al., 2019; Fookes et al., 618 2011; Shah et al., 2005). Most of the representative subset of TN isolates analyzed for SPI 619 contained SPI-2 and SPI-4. SPI-2 encodes a type III secretion system 1 (TTSS-2), which is 620 involved in intracellular survival and replication, immune evasion, and systemic pathogenicity 621 (Schmidt \& Hensel, 2004; Tsai \& Coombes, 2019), replication within macrophages, and 622 systemic infections (Jajere, 2019). SPI-4 encodes genes for toxin secretion and apoptosis and is 623 involved in intracellular (macrophage) survival (Jajere, 2019). All three genes associated with 624 the cytolethal distending toxin were identified in all of the representative subset of TN isolates,

625

626

627

628

629

630

631

632

633

634

635

636

637

638

639

640

641

642

643

644

645

646

647

648

649

650

651

652

653

654 which is in agreement with other studies that have identified these three genes in all Salmonella ser. Javiana isolates tested (Mezal et al., 2013; Miller \& Wiedmann, 2016). Other virulence genes that differed among isolates were mainly associated with mobile genetic elements.

All 111 TN Salmonella ser. Javiana isolates analyzed in the present study contained the $a a c\left(6^{\prime}\right)$-Iaa gene, which is associated with aminoglycoside resistance (Shaw et al., 1993).

However, there is evidence that this gene is cryptic and no longer confers phenotypic aminoglycoside resistance (Leon et al., 2018; Salipante \& Hall, 2003), which is consistent with the low prevalence of phenotypic resistance to amikacin and gentamicin (0.04\%) in U.S. clinical Salmonella ser. Javiana isolates (Centers for Disease Control and Prevention (CDC), 2019b). Taken together, these findings highlight the complexity of antimicrobial resistance. The three other antibiotic resistance genes identified in this study ( $a p h\left(3^{\prime}\right)-I a$, sul3, and qnrB19 gene) were each only present in a single isolate. The low prevalence of these three genes is consistent with the low phenotypic prevalence of resistance to gentamicin and kanamycin $(0.12 \%)$, sulfamethoxazole/sulfisoxazole $(0.63 \%)$, trimethoprim-sulfamethoxazole $(0.21 \%)$, and ciprofloxacin (0\%) seen in U.S. clinical Salmonella ser. Javiana isolates (Centers for Disease Control and Prevention (CDC), 2019b). Additionally, The hypothesis that the qnrB19 gene may not be functional is further supported by the fact that phenotypic ciprofloxacin resistance has not been reported in Salmonella ser. Javiana clinical isolates (Centers for Disease Control and Prevention (CDC), 2019b). As aminoglycosides are not typically used to treat Salmonella infections, the presence of the $a a c\left(6^{\prime}\right)$-Iaa and $a p h\left(3^{\prime}\right)$-Ia genes is of little clinical significance. Overall, these data show a low prevalence of genes associated antibiotic resistance in Salmonella ser. Javiana from TN. However, antibiotic susceptibility testing would need to be performed on these isolates to confirm.

\section{Conclusions}

This study demonstrates the population structure of Salmonella ser. Javiana in Tennessee and globally. As this is a clinically important Salmonella serotype, understanding the phylogeny can provide guidance for phylogenetic analyses and cluster detection for public health 
655 surveillance and response. We show that Salmonella ser. Javiana clinical isolates from TN show 656 geospatial and temporal distribution, with most isolates originating from the western part of the 657 state and during the summer months (July, August, and September). Based on the results of the 658 pan-GWAS, it is clear that MGEs (namely plasmids and prophage regions) in the genome 659 account for most of the differences in gene content between the three main clades of this serovar. 660 This is noteworthy, as clinically-relevant genes (like ABR-conferring or virulence-related genes) 661 can be found in these regions and they could potentially be used for isolate characterization.

662 Additionally, we found that when performing hqSNP analysis for epidemiological cluster 663 detection with the TN isolates, it is not necessary to first divide the isolates into clades, as we 664 found this only minimally increases the SNP differences between isolates; however the TN 665 isolates all belonged to a single global major clade and single ceBG, so this may only be 666 applicable to less diverse populations. Further research should include clinical Salmonella ser. 667 Javiana isolates and associated metadata from other states to obtain a more complete 668 representation of the population structure of and epidemiological information about Salmonella 669 ser. Javiana in the United States and an analysis of disease severity and gene content could assist 670 in the identification of genes that may be involved in virulence. Another research direction 671 would be to include isolates from other sources (i.e., environmental, animal, food) in a 672 phylogenetic analysis, which may expand our understanding of the population structure and 673 including isolates with diverse isolation sources may provide insight into source attribution and 674 potential recommendations to prevent morbidity. 
Achtman M, Wain J, Weill F-X, Nair S, Zhou Z, Sangal V, Krauland MG, Hale JL, Harbottle H,

678

679

680

681

682

683

684

685

686

687

688

689

690

691

692

693

694

695

696

697

698

699

700

701

702

703

704

705

706

707

708

709

710

711

712

713

714

715

716

717

718

719 Uesbeck A, Dougan G, Harrison LH, Brisse S, and the SeMsg. 2012. Multilocus sequence typing as a replacement for serotyping in Salmonella enterica. PLOS Pathogens 8:e1002776. 10.1371/journal.ppat.1002776

Adesiyun AA, Campbell M, and Kaminjolo JS. 1997. Prevalence of bacterial enteropathogens in pet dogs in Trinidad. Journal of Veterinary Medicine, Series B 44:19-27. 10.1111/j.14390450.1997.tb00946.x

Adesiyun AA, Webb LA, Romain H, and Kaminjolo JS. 1996. Prevalence of Salmonella, Listeria monocytogenes, Campylobacter spp., Yersinia enterocolitica and Cryptosporidium spp. In bulk milk, cows' faeces and effluents of dairy farms in Trinidad. Revue d'elevage et de medecine veterinaire des pays tropicaux 49:303-309.

Alikhan NF, Zhou ZM, Sergeant MJ, and Achtman M. 2018. A genomic overview of the population structure of Salmonella. PLOS Genetics 14:13. 10.1371/journal.pgen.1007261

Alix E, and Blanc-Potard A-B. 2009. Hydrophobic peptides: Novel regulators within bacterial membrane. Molecular Microbiology 72:5-11. 10.1111/j.1365-2958.2009.06626.x

Alley RD, and Pijoan M. 1942. Salmonella Javiana food infection. The Yale Journal of Biology and Medicine 15:229-239.

Andrews S. 2010. FastQC. Available at https://www.bioinformatics.babraham.ac.uk/projects/fastqc/ (accessed 11 March 2019).

Antipov D, Hartwick N, Shen M, Raiko M, Lapidus A, and Pevzner PA. 2016. plasmidSPAdes: Assembling plasmids from whole genome sequencing data. Bioinformatics 32:33803387. 10.1093/bioinformatics/btw493

Arndt D, Grant JR, Marcu A, Sajed T, Pon A, Liang Y, and Wishart DS. 2016. PHASTER: A better, faster version of the PHAST phage search tool. Nucleic Acids Research 44:W1621. 10.1093/nar/gkw387

Ashton PM, Nair S, Peters TM, Bale JA, Powell DG, Painset A, Tewolde R, Schaefer U, Jenkins C, and Dallman TJ. 2016. Identification of Salmonella for public health surveillance using whole genome sequencing. PeerJ 4:e1752.

Banerji S, Simon S, Tille A, Fruth A, and Flieger A. 2020. Genome-based Salmonella serotyping as the new gold standard. Scientific Reports 10:4333. 10.1038/s41598-020-61254-1

Bankevich A, Nurk S, Antipov D, Gurevich AA, Dvorkin M, Kulikov AS, Lesin VM, Nikolenko SI, Pham S, Prjibelski AD, Pyshkin AV, Sirotkin AV, Vyahhi N, Tesler G, Alekseyev MA, and Pevzner PA. 2012. SPAdes: A new genome assembly algorithm and its applications to single-cell sequencing. Journal of Computational Biology 19:455-477. 10.1089/cmb.2012.0021

Bäumler AJ, Tsolis RM, Bowe FA, Kusters JG, Hoffmann S, and Heffron F. 1996. The pef fimbrial operon of Salmonella Typhimurium mediates adhesion to murine small intestine and is necessary for fluid accumulation in the infant mouse. Infection and Immunity 64:61-68.

Bell RL, Zheng J, Burrows E, Allard S, Wang CY, Keys CE, Melka DC, Strain E, Luo Y, Allard MW, Rideout S, and Brown EW. 2015. Ecological prevalence, genetic diversity, and epidemiological aspects of Salmonella isolated from tomato agricultural regions of the Virginia eastern shore. Frontiers in Microbiology 6:15. 10.3389/fmicp.2015.00415 
720

721

722

723

724

725

726

727

728

729

730

731

732

733

734

735

736

737

738

739

740

741

742

743

744

745

746

747

748

749

750

751

752

753

754

755

756

757

758

759

760

761

762

763

Bennett SD, Littrell KW, Hill TA, Mahovic M, and Behravesh CB. 2015. Multistate foodborne disease outbreaks associated with raw tomatoes, United States, 1990-2010: A recurring public health problem. Epidemiology and Infection 143:1352-1359. 10.1017/S0950268814002167

Besser JM, Carleton HA, Trees E, Stroika SG, Hise K, Wise M, and Gerner-Smidt P. 2019. Interpretation of whole-genome sequencing for enteric disease surveillance and outbreak investigation. Foodborne Pathogens and Disease 16:504-512. 10.1089/fpd.2019.2650

Blostein J. 1993. An outbreak of Salmonella Javiana associated with consumption of watermelon. Journal of Environmental Health 56:29-31.

Bolger AM, Lohse M, and Usadel B. 2014. Trimmomatic: A flexible trimmer for Illumina sequence data. Bioinformatics 30:2114-2120.

Boore AL, Hoekstra RM, Iwamoto M, Fields PI, Bishop RD, and Swerdlow DL. 2015. Salmonella enterica infections in the United States and assessment of coefficients of variation: A novel approach to identify epidemiologic characteristics of individual serotypes, 1996-2011. PLOS ONE 10:e0145416-e0145416.

10.1371/journal.pone.0145416

Brar PK, Strawn LK, and Danyluk MD. 2016. Prevalence, level, and types of Salmonella isolated from North American in-shell pecans over four harvest years. Journal of Food Protection 79:352-360. 10.4315/0362-028x.Jfp-15-365

Brettin T, Davis JJ, Disz T, Edwards RA, Gerdes S, Olsen GJ, Olson R, Overbeek R, Parrello B, Pusch GD, Shukla M, Thomason JA, 3rd, Stevens R, Vonstein V, Wattam AR, and Xia F. 2015. RASTtk: A modular and extensible implementation of the RAST algorithm for building custom annotation pipelines and annotating batches of genomes. Scientific Reports 5:8365-8365. 10.1038/srep08365

Brynildsrud O, Bohlin J, Scheffer L, and Eldholm V. 2016. Rapid scoring of genes in microbial pan-genome-wide association studies with Scoary. Genome Biology 17:238. 10.1186/s13059-016-1108-8

Bushnell B. 2018. Bbtools: A suite of fast, multithreaded bioinformatics tools designed for analysis of DNA and RNA sequence data. Available at https://jgi.doe.gov/data-andtools/bbtools/ (accessed 25 June 2019).

Cao G, Meng J, Strain E, Stones R, Pettengill J, Zhao S, McDermott P, Brown E, and Allard M. 2013. Phylogenetics and differentiation of Salmonella Newport lineages by whole genome sequencing. PLOS ONE 8:e55687. 10.1371/journal.pone.0055687

Carattoli A, Zankari E, García-Fernández A, Voldby Larsen M, Lund O, Villa L, Møller Aarestrup F, and Hasman H. 2014. In silico detection and typing of plasmids using PlasmidFinder and plasmid multilocus sequence typing. Antimicrobial Agents and Chemotherapy 58:3895-3903. 10.1128/aac.02412-14

Carniel E. 2001. The Yersinia high-pathogenicity island: An iron-uptake island. Microbes and Infection 3:561-569. https://doi.org/10.1016/S1286-4579(01)01412-5

Casas MRT, Camargo CH, Soares FB, da Silveira WD, and Fernandes SA. 2016. Presence of plasmid-mediated quinolone resistance determinants and mutations in gyrase and topoisomerase in Salmonella enterica isolates with resistance and reduced susceptibility to ciprofloxacin. Diagnostic Microbiology and Infectious Disease 85:85-89. https://doi.org/10.1016/j.diagmicrobio.2016.01.016 
764

765

766

767

768

769

770

771

772

773

774

775

776

777

778

779

780

781

782

783

784

785

786

787

788

789

790

791

792

793

794

795

796

797

798

799

800

801

802

803

804

805

806

807

808

Centers for Disease Control and Prevention (CDC). 2002. Multistate outbreaks of Salmonella serotype Poona infections associated with eating cantaloupe from Mexico--United States and Canada, 2000-2002. MMWR Morbidity and Mortality Weekly Report 51:1044.

Centers for Disease Control and Prevention (CDC). 2005. Outbreaks of Salmonella infections associated with eating roma tomatoes--United States and Canada, 2004. MMWR Morbidity and Mortality Weekly Report 54:325.

Centers for Disease Control and Prevention (CDC). 2007. Multistate outbreaks of Salmonella infections associated with raw tomatoes eaten in restaurants--United States, 2005-2006. MMWR Morbidity and Mortality Weekly Report 56:909-911.

Centers for Disease Control and Prevention (CDC). 2013. An atlas of Salmonella in the United States, 1968-2011: Laboratory-based enteric disease surveillance (serotype Javiana). Available at https://www.cdc.gov/salmonella/pdf/javiana-508c.pdf (accessed 15 May 2019).

Centers for Disease Control and Prevention (CDC). 2016. PulseNet methods \& protocols: WGS. Available at https://www.cdc.gov/pulsenet/pathogens/wgs.html (accessed 2020 July 22 2020).

Centers for Disease Control and Prevention (CDC). 2017a. Foodborne Diseases Active Surveillance Network (FoodNet): FoodNet 2015 surveillance report (final data). In: U.S. Department of Health and Human Services C, editor. Atlanta, Georgia.

Centers for Disease Control and Prevention (CDC). 2017b. Standard operating procedure for PulseNet PFGE of Escherichia coli O157:H7, Escherichia coli non-O157 (STEC), Salmonella serotypes, Shigella sonnei and Shigella flexneri. Available at https://www.cdc.gov/pulsenet/pdf/ecoli-shigella-salmonella-pfge-protocol-508c.pdf (accessed 7 May 2020).

Centers for Disease Control and Prevention (CDC). 2018. National Salmonella surveillance annual report, 2016.

Centers for Disease Control and Prevention (CDC). 2019a. Antibiotic resistance threats in the United States, 2019. U.S. Department of Health and Human Services, CDC.

Centers for Disease Control and Prevention (CDC). 2019b. National antimicrobial resistance monitoring system (NARMS) now: Human data. Available at https://www.cdc.gov/narmsnow (accessed 22 November 2019).

Cheng RA, Eade CR, and Wiedmann M. 2019. Embracing diversity: Differences in virulence mechanisms, disease severity, and host adaptations contribute to the success of nontyphoidal Salmonella as a foodborne pathogen. Frontiers in Microbiology 10:13681368. 10.3389/fmicb.2019.01368

Clarkson LS, Tobin-D'Angelo M, Shuler C, Hanna S, Benson J, and Voetsch AC. 2010. Sporadic Salmonella enterica serotype Javiana infections in Georgia and Tennessee: A hypothesisgenerating study. Epidemiology and Infection 138:340-346.

Correia S, Poeta P, Hébraud M, Capelo JL, and Igrejas G. 2017. Mechanisms of quinolone action and resistance: Where do we stand? Journal of Medical Microbiology 66:551-559. https://doi.org/10.1099/jmm.0.000475

Crump JA, Sjölund-Karlsson M, Gordon MA, and Parry CM. 2015. Epidemiology, clinical presentation, laboratory diagnosis, antimicrobial resistance, and antimicrobial management of invasive Salmonella infections. Clinical Microbiology Reviews 28:901937. $10.1128 / \mathrm{cmr} .00002-15$

Peer) reviewing PDF | (2020:05:49215:2:0:NEW 30 Sep 2020) 
809 Cuypers WL, Jacobs J, Wong V, Klemm EJ, Deborggraeve S, and Van Puyvelde S. 2018.

810

811

812

813

814

815

816

817

818

819

820

821

822

823

824

825

826

827

828

829

830

831

832

833

834

835

836

837

838

839

840

841

842

843

844

845

846

847

848

849

850

851

852

853

854
Fluoroquinolone resistance in Salmonella: Insights by whole-genome sequencing. Microbial Genomics 4:9. 10.1099/mgen.0.000195

Davis S, Pettengill JB, Luo Y, Payne J, Shpuntoff A, Rand H, and Strain E. 2015. CFSAN SNP pipeline: An automated method for constructing SNP matrices from next-generation sequence data. PeerJ Computer Science 1:e20.

den Bakker HC, Moreno Switt AI, Govoni G, Cummings CA, Ranieri ML, Degoricija L, Hoelzer K, Rodriguez-Rivera LD, Brown S, Bolchacova E, Furtado MR, and Wiedmann M. 2011. Genome sequencing reveals diversification of virulence factor content and possible host adaptation in distinct subpopulations of Salmonella enterica. BMC Genomics 12:425425. 10.1186/1471-2164-12-425

Drake M, Amadi V, Zieger U, Johnson R, and Hariharan H. 2013. Prevalence of Salmonella spp. In cane toads (bufo marinus) from Grenada, West Indies, and their antimicrobial susceptibility. Zoonoses and Public Health 60:437-441. doi:10.1111/zph.12018

Duffy EA, Lucia LM, Kells JM, Castillo A, Pillai SD, and Acuff GR. 2005. Concentrations of Escherichia coli and genetic diversity and antibiotic resistance profiling of Salmonella isolated from irrigation water, packing shed equipment, and fresh produce in Texas. Journal of Food Protection 68:70-79. 10.4315/0362-028x-68.1.70

Elder JR, Chiok KL, Paul NC, Haldorson G, Guard J, and Shah DH. 2016. The Salmonella pathogenicity island 13 contributes to pathogenesis in streptomycin pre-treated mice but not in day-old chickens. Gut Pathogens 8:16. 10.1186/s13099-016-0098-0

Elviss NC, Little CL, Hucklesby L, Sagoo S, Surman-Lee S, de Pinna E, and Threlfall EJ. 2009. Microbiological study of fresh herbs from retail premises uncovers an international outbreak of salmonellosis. International Journal of Food Microbiology 134:83-88. https://doi.org/10.1016/j.ijfoodmicro.2009.01.015

Elward A, Grim A, Schroeder P, Kieffer P, Sellenriek P, Ferrett R, Adams H, xa, Chaski, Phillips V, Bartow R, Mays D, Lawrence S, Seed P, Holzmann, x, Pazgal G, Polish L, Leet T, and Fraser V. 2006. Outbreak of Salmonella Javiana infection at a children's hospital. Infection Control and Hospital Epidemiology 27:586-592. 10.1086/506483

Eng S-K, Pusparajah P, Ab Mutalib N-S, Ser H-L, Chan K-G, and Lee L-H. 2015. Salmonella: A review on pathogenesis, epidemiology and antibiotic resistance. Frontiers in Life Science 8:284-293.

EnteroBase Team. 2018. Enterobase: HierCC equivalents. Available at https://enterobase.readthedocs.io/en/latest/HierCC lookup.html (accessed 31 July 2020).

Espinoza RA, Silva Valenzuela CA, Amaya FA, Urrutia ÍM, Contreras I, and Santiviago CA. 2017. Differential roles for pathogenicity islands SPI-13 and SPI-8 in the interaction of Salmonella Enteritidis and Salmonella Typhi with murine and human macrophages. Biological Research 50.

Ewels P, Magnusson M, Lundin S, and Käller M. 2016. MultiQC: Summarize analysis results for multiple tools and samples in a single report. Bioinformatics 32:3047-3048. 10.1093/bioinformatics/btw354

Felsenstein J. 1985. Confidence limits on phylogenies: An approach using the bootstrap. Evolution 39:783-791. 10.1111/j.1558-5646.1985.tb00420.x

Filloux A, Michel G, and Bally M. 1998. GSP-dependent protein secretion in Gram-negative bacteria: The Xcp system of Pseudomonas aeruginosa. Fems Microbiology Reviews 22:177-198. 10.1111/j.1574-6976.1998.tb00366.x 
855

856

857

858

859

860

861

862

863

864

865

866

867

868

869

870

871

872

873

874

875

876

877

878

879

880

881

882

883

884

885

886

887

888

889

890

891

892

893

894

895

896

897

898
Folkesson A, Advani A, Sukupolvi S, Pfeifer JD, Normark S, and Löfdahl S. 1999. Multiple insertions of fimbrial operons correlate with the evolution of Salmonella serovars responsible for human disease. Molecular Microbiology 33:612-622. 10.1046/j.13652958.1999.01508.x

Fookes M, Schroeder GN, Langridge GC, Blondel CJ, Mammina C, Connor TR, Seth-Smith H, Vernikos GS, Robinson KS, Sanders M, Petty NK, Kingsley RA, Bäumler AJ, Nuccio SP, Contreras I, Santiviago CA, Maskell D, Barrow P, Humphrey T, Nastasi A, Roberts M, Frankel G, Parkhill J, Dougan G, and Thomson NR. 2011. Salmonella bongori provides insights into the evolution of the salmonellae. PLOS Pathogens 7:e1002191. 10.1371/journal.ppat.1002191

Fozo EM, Kawano M, Fontaine F, Kaya Y, Mendieta KS, Jones KL, Ocampo A, Rudd KE, and Storz G. 2008. Repression of small toxic protein synthesis by the Sib and OhsC small RNAs. Molecular Microbiology 70:1076-1093. 10.1111/j.1365-2958.2008.06394.x

Galata V, Fehlmann T, Backes C, and Keller A. 2018. Plsdb: A resource of complete bacterial plasmids. Nucleic Acids Research 47:D195-D202. 10.1093/nar/gky1050

Gama N, Berchieri Jr A, and Fernandes S. 2003. Occurrence of Salmonella sp in laying hens. Brazilian Journal of Poultry Science 5:15-21.

Gardner SN, Slezak T, and Hall BG. 2015. Ksnp3.0: SNP detection and phylogenetic analysis of genomes without genome alignment or reference genome. Bioinformatics 31:2877-2878.

Gilbert DN, Chambers HF, Eliopoulos GM, and Saag MS. 2016. The Sanford guide to antimicrobial therapy 2016. 46th edition.. ed: Sperryville, Va. : Antimicrobial Therapy.

Gopee NV, Adesiyun AA, and Caesar K. 2000. Retrospective and longitudinal study of salmonellosis in captive wildlife in Trinidad. Journal of Wildlife Diseases 36:284-293. 10.7589/0090-3558-36.2.284

Gruszynski K, Pao S, Kim C, Toney D, Wright K, Ross PG, Colon A, and Levine S. 2014. Evaluating wildlife as a potential source of Salmonella serotype Newport (jjpx01.0061) contamination for tomatoes on the eastern shore of Virginia. Zoonoses and Public Health 61:202-207. 10.1111/zph.12061

Gurevich A, Saveliev V, Vyahhi N, and Tesler G. 2013. QUAST: Quality assessment tool for genome assemblies. Bioinformatics 29:1072-1075. 10.1093/bioinformatics/btt086

Hanning IB, Nutt J, and Ricke SC. 2009. Salmonellosis outbreaks in the United States due to fresh produce: Sources and potential intervention measures. Foodborne Pathogens and Disease 6:635-648.

Harris CS, Tertuliano M, Rajeev S, Vellidis G, and Levy K. 2018. Impact of storm runoff on Salmonella and Escherichia coli prevalence in irrigation ponds of fresh produce farms in southern Georgia. Journal of Applied Microbiology 124:910-921. 10.1111/jam.13689

Hedberg CW, Korlath JA, D'Aoust JY, White KE, Schell WL, Miller MR, Cameron DN, MacDonald KL, and Osterholm MT. 1992. A multistate outbreak of Salmonella Javiana and Salmonella Oranienburg infections due to consumption of contaminated cheese. Journal of the American Medical Association 268:3203-3207.

Huang JY, Patrick ME, Manners J, Sapkota AR, Scherzinger KJ, Tobin-D'Angelo M, Henao OL, Cole DJ, and Vieira AR. 2017. Association between wetland presence and incidence of Salmonella enterica serotype Javiana infections in selected US sites, 2005-2011. Epidemiology and Infection 145:2991-2997. 10.1017/S0950268817001790 
899

900

901

902

903

904

905

906

907

908

909

910

911

912

913

914

915

916

917

918

919

920

921

922

923

924

925

926

927

928

929

930

931

932

933

934

935

936

937

938

939

940

941

942

943

944

Iveson JB, and Bradshaw SD. 1973. Salmonella Javiana infection in an infant associated with a marsupial, quokka, setonix brachyurus, in western Australia. Journal of Hygiene 71:423432. 10.1017/s0022172400046404

Jackson BR, Griffin PM, Cole D, Walsh KA, and Chai SJ. 2013. Outbreak-associated Salmonella enterica serotypes and food commodities, United States, 1998-2008. Emerging Infectious Diseases 19:1239-1244. 10.3201/eid1908.121511

Jacobsen A, Hendriksen RS, Aaresturp FM, Ussery DW, and Friis C. 2011. The Salmonella enterica pan-genome. Microbial Ecology 62:487. 10.1007/s00248-011-9880-1

Jagadeesan B, Baert L, Wiedmann M, and Orsi RH. 2019. Comparative analysis of tools and approaches for source tracking Listeria monocytogenes in a food facility using wholegenome sequence data. Frontiers in Microbiology 10. 10.3389/fmicb.2019.00947

Jajere SM. 2019. A review of Salmonella enterica with particular focus on the pathogenicity and virulence factors, host specificity and antimicrobial resistance including multidrug resistance. Veterinary World 12:504-521. 10.14202/vetworld.2019.504-521

Joensen KG, Scheutz F, Lund O, Hasman H, Kaas RS, Nielsen EM, and Aarestrup FM. 2014. Real-time whole-genome sequencing for routine typing, surveillance, and outbreak detection of verotoxigenic Escherichia coli. Journal of Clinical Microbiology 52:15011510. 10.1128/jcm.03617-13

Jones TF, Ingram LA, Cieslak PR, Vugia DJ, Tobin-D'Angelo M, Hurd S, Medus C, Cronquist A, and Angulo FJ. 2008. Salmonellosis outcomes differ substantially by serotype. The Journal of Infectious Diseases 198:109-114. 10.1086/588823

Katz LS, Griswold T, Williams-Newkirk AJ, Wagner D, Petkau A, Sieffert C, Van Domselaar G, Deng X, and Carleton HA. 2017. A comparative analysis of the Lyve-SET phylogenomics pipeline for genomic epidemiology of foodborne pathogens. Frontiers in Microbiology 8. 10.3389/fmicb.2017.00375

Kawano M, Oshima T, Kasai H, and Mori H. 2002. Molecular characterization of long direct repeat (LDR) sequences expressing a stable mRNA encoding for a 35-amino-acid cellkilling peptide and a cis-encoded small antisense RNA in Escherichia coli. Molecular Microbiology 45:333-349. 10.1046/j.1365-2958.2002.03042.x

Kumar S, Stecher G, and Tamura K. 2016. MEGA7: Molecular evolutionary genetics analysis version 7.0 for bigger datasets. Molecular Biology and Evolution 33:1870-1874.

Leahy AM, Cummings KJ, Rodriguez-Rivera LD, Rankin SC, and Hamer SA. 2016. Evaluation of faecal Salmonella shedding among dogs at seven animal shelters across Texas. Zoonoses and Public Health 63:515-521. 10.1111/zph.12257

Lee R, Peppe J, and George H. 1998. Pulsed-field gel electrophoresis of genomic digests demonstrates linkages among food, food handlers, and patrons in a food-borne Salmonella Javiana outbreak in Massachusetts. Journal of Clinical Microbiology 36:284285.

Leekitcharoenphon P, Nielsen EM, Kaas RS, Lund O, and Aarestrup FM. 2014. Evaluation of whole genome sequencing for outbreak detection of Salmonella enterica. PLOS ONE 9:e87991.

Lehmacher A, Bockemühl J, and Aleksic S. 1995. Nationwide outbreak of human salmonellosis in Germany due to contaminated paprika and paprika-powdered potato chips.

Epidemiology and Infection 115:501-511. 10.1017/S0950268800058660

Leon IM, Lawhon SD, Norman KN, Threadgill DS, Ohta N, Vinasco J, and Scott HM. 2018. Serotype diversity and antimicrobial resistance among Salmonella enterica isolates from

Peer) reviewing PDF | (2020:05:49215:2:0:NEW 30 Sep 2020) 
945

946

947

948

949

950

951

952

953

954

955

956

957

958

959

960

961

962

963

964

965

966

967

968

969

970

971

972

973

974

975

976

977

978

979

980

981

982

983

984

985

986

987

988

989

990

patients at an equine referral hospital. Applied and Environmental Microbiology 84:e02829-02817. 10.1128/aem.02829-17

Letunic I, and Bork P. 2016. Interactive tree of life (iTOL) v3: An online tool for the display and annotation of phylogenetic and other trees. Nucleic Acids Research 44:W242-245. $10.1093 / \mathrm{nar} / \mathrm{gkw} 290$

Li BG, Vellidis G, Liu HL, Jay-Russell M, Zhao SH, Hu ZL, Wright A, and Elkins CA. 2014. Diversity and antimicrobial resistance of Salmonella enterica isolates from surface water in southeastern United States. Applied and Environmental Microbiology 80:6355-6365. 10.1128/aem.02063-14

Li H, Handsaker B, Wysoker A, Fennell T, Ruan J, Homer N, Marth G, Abecasis G, Durbin R, and Genome Project Data Processing S. 2009. The sequence alignment/map format and SAMtools. Bioinformatics 25:2078-2079. 10.1093/bioinformatics/btp352

Liu B, Zheng D, Jin Q, Chen L, and Yang J. 2018. VFDB 2019: A comparative pathogenomic platform with an interactive web interface. Nucleic Acids Research 47:D687-D692. 10.1093/nar/gky1080

Lockhart JM, Lee G, Turco J, and Chamberlin L. 2008. Salmonella from gopher tortoises (gopherus polyphemus) in south Georgia. Journal of Wildlife Diseases 44:988-991. 10.7589/0090-3558-44.4.988

Mezal EH, Stefanova R, and Khan AA. 2013. Isolation and molecular characterization of Salmonella enterica serovar Javiana from food, environmental and clinical samples. International Journal of Food Microbiology 164:113-118. https://doi.org/10.1016/j.ijfoodmicro.2013.03.021

Micallef SA, Goldstein RER, George A, Kleinfelter L, Boyer MS, McLaughlin CR, Estrin A, Ewing L, Beaubrun JJG, Hanes DE, Kothary MH, Tall BD, Razeq JH, Joseph SW, and Sapkota AR. 2012. Occurrence and antibiotic resistance of multiple Salmonella serotypes recovered from water, sediment and soil on mid-atlantic tomato farms. Environmental Research 114:31-39. 10.1016/j.envres.2012.02.005

Miller RA, Betteken MI, Guo XD, Altier C, Duhamel GE, and Wiedmann M. 2018. The typhoid toxin produced by the nontyphoidal Salmonella enterica serotype Javiana is required for induction of a DNA damage response in vitro and systemic spread in vivo. mBio 9:16. 10.1128/mBio.00467-18

Miller RA, and Wiedmann M. 2016. The cytolethal distending toxin produced by nontyphoidal Salmonella serotypes Javiana, Montevideo, Oranienburg, and Mississippi induces DNA damage in a manner similar to that of serotype Typhi. mBio 7:11. 10.1128/mBio.0210916

Miller S, Amadi V, Stone D, Johnson R, Hariharan H, and Zieger U. 2014. Prevalence and antimicrobial susceptibility of Salmonella spp. In small Indian mongooses (herpestes auropunctatus) in Grenada, West Indies. Comparative Immunology, Microbiology and Infectious Diseases 37:205-210. https://doi.org/10.1016/j.cimid.2014.05.003

Mukherjee N, Nolan VG, Dunn JR, and Banerjee P. 2019. Sources of human infection by Salmonella enterica serotype Javiana: A systematic review. PLOS ONE 14:e222108. 10.1371/journal.pone.0222108

Mukherjee N, Nolan VG, Dunn JR, and Banerjee P. 2020. Exposures associated with nontyphoidal Salmonella infections caused by Newport, Javiana, and Mississippi serotypes in Tennessee, 2013-2015: A case-case analysis. Pathogens 9:78.

Nei M, and Kumar S. 2000. Molecular evolution and phylogenetics: Oxford university press. 
991 Oliveira CJB, Carvalho LFOS, Fernandes SA, Tavechio AT, Menezes CCP, and Domingues Jr

992

993

994

995

996

997

998

999

1000

1001

1002

1003

1004

1005

1006

1007

1008

1009

1010

1011

1012

1013

1014

1015

1016

1017

1018

1019

1020

1021

1022

1023

1024

1025

1026

1027

1028

1029

1030

1031

1032

1033

1034

1035
FJ. 2002. Antimicrobial resistance of Salmonella serotypes isolated from slaughter-age pigs and environmental samples. Microbial Drug Resistance 8:407-411. $10.1089 / 10766290260469697$

Page AJ, Cummins CA, Hunt M, Wong VK, Reuter S, Holden MTG, Fookes M, Falush D, Keane JA, and Parkhill J. 2015. Roary: Rapid large-scale prokaryote pan genome analysis. Bioinformatics 31:3691-3693. 10.1093/bioinformatics/btv421

Perreten V, and Boerlin P. 2003. A new sulfonamide resistance gene sul3 in Escherichia coli is widespread in the pig population of Switzerland. Antimicrobial Agents and Chemotherapy 47:1169-1172. 10.1128/aac.47.3.1169-1172.2003

Portmann A-C, Fournier C, Gimonet J, Ngom-Bru C, Barretto C, and Baert L. 2018. A validation approach of an end-to-end whole genome sequencing workflow for source tracking of Listeria monocytogenes and Salmonella enterica. Frontiers in Microbiology 9. 10.3389/fmicb.2018.00446

Raspoet R, Eeckhaut V, Vermeulen K, De Smet L, Wen Y, Nishino K, Haesebrouck F, Ducatelle R, Devreese B, and Van Immerseel F. 2019. The Salmonella Enteritidis TolC outer membrane channel is essential for egg white survival. Poultry Science 98:2281-2289. $10.3382 / \mathrm{ps} / \mathrm{pey} 584$

Reddy SP, Wang H, Adams JK, and Feng PCH. 2016. Prevalence and characteristics of Salmonella serotypes isolated from fresh produce marketed in the United States. Journal of Food Protection 79:6-16. 10.4315/0362-028x.Jfp-15-274

Redgrave LS, Sutton SB, Webber MA, and Piddock LJV. 2014. Fluoroquinolone resistance: Mechanisms, impact on bacteria, and role in evolutionary success. Trends in Microbiology 22:438-445. https://doi.org/10.1016/j.tim.2014.04.007

Rodriguez A, Pangloli P, Richards HA, Mount JR, and Draughton FA. 2006. Prevalence of Salmonella in diverse environmental farm samples. Journal of Food Protection 69:25762580.

Roer L, Hendriksen RS, Leekitcharoenphon P, Lukjancenko O, Kaas RS, Hasman H, and Aarestrup FM. 2016. Is the evolution of Salmonella enterica subsp. enterica linked to restriction-modification systems? mSystems 1:e00009-00016. 10.1128/mSystems.0000916

Saitou N, and Nei M. 1987. The neighbor-joining method: A new method for reconstructing phylogenetic trees. Molecular Biology and Evolution 4:406-425.

10.1093/oxfordjournals.molbev.a040454

Salipante SJ, and Hall BG. 2003. Determining the limits of the evolutionary potential of an antibiotic resistance gene. Molecular Biology and Evolution 20:653-659. 10.1093/molbev/msg074

Sandt CH, Krouse DA, Cook CR, Hackman AL, Chmielecki WA, and Warren NG. 2006. The key role of pulsed-field gel electrophoresis in investigation of a large multiserotype and multistate food-borne outbreak of Salmonella infections centered in Pennsylvania. Journal of Clinical Microbiology 44:3208-3212. 10.1128/jcm.01404-06

Sangal V, Harbottle H, Mazzoni CJ, Helmuth R, Guerra B, Didelot X, Paglietti B, Rabsch W, Brisse S, Weill F-X, Roumagnac P, and Achtman M. 2010. Evolution and population structure of Salmonella enterica serovar Newport. Journal of Bacteriology 192:64656476. 10.1128/jb.00969-10

Peer] reviewing PDF | (2020:05:49215:2:0:NEW 30 Sep 2020) 
1036

1037

1038

1039

1040

1041

1042

1043

1044

1045

1046

1047

1048

1049

1050

1051

1052

1053

1054

1055

1056

1057

1058

1059

1060

1061

1062

1063

1064

1065

1066

1067

1068

1069

1070

1071

1072

1073

1074

1075

1076

1077

1078

1079

1080

1081

Santos FBO, D'Souza DH, Jaykus L, Ferket PR, and Sheldon BW. 2007. Genotypes, serotypes, and antibiotic resistance profiles of Salmonella isolated from commercial North Carolina turkey farms. Journal of Food Protection 70:1328-1333. 10.4315/0362-028x-70.6.1328

Scallan E, Hoekstra RM, Angulo FJ, Tauxe RV, Widdowson M-A, Roy SL, Jones JL, and Griffin PM. 2011. Foodborne illness acquired in the United States--major pathogens. Emerging Infectious Diseases 17:7-15. 10.3201/eid1701.P11101

Schmidt H, and Hensel M. 2004. Pathogenicity islands in bacterial pathogenesis. Clinical Microbiology Reviews 17:14-56. 10.1128/cmr.17.1.14-56.2004

Seemann T. 2014. Prokka: Rapid prokaryotic genome annotation. Bioinformatics 30:2068-2069. 10.1093/bioinformatics/btu153

Seepersadsingh N, Adesiyun AA, and Seebaransingh R. 2004. Prevalence and antimicrobial resistance of Salmonella spp. In non-diarrhoeic dogs in Trinidad. Journal of Veterinary Medicine B, Infectious Diseases and Veterinary Public Health 51:337-342. 10.1111/j.1439-0450.2004.00785.x

Sévellec Y, Vignaud M-L, Granier SA, Lailler R, Feurer C, Le Hello S, Mistou M-Y, and CadelSix S. 2018. Polyphyletic nature of Salmonella enterica serotype Derby and lineagespecific host-association revealed by genome-wide analysis. Frontiers in Microbiology 9. 10.3389/fmicb.2018.00891

Shah DH, Lee M-j, Park J-h, Lee J-h, Eo S-k, Kwon J-t, and Chae J-s. 2005. Identification of Salmonella Gallinarum virulence genes in a chicken infection model using PCR-based signature-tagged mutagenesis. Microbiology 151:3957-3968. https://doi.org/10.1099/mic.0.28126-0

Shane AL, Mody RK, Crump JA, Tarr PI, Steiner TS, Kotloff K, Langley JM, Wanke C, Warren CA, Cheng AC, Cantey J, and Pickering LK. 2017. 2017 Infectious Diseases Society of America clinical practice guidelines for the diagnosis and management of infectious diarrhea. Clinical Infectious Diseases 65:e45-e80. 10.1093/cid/cix669

Shaw KJ, Rather PN, Hare RS, and Miller GH. 1993. Molecular genetics of aminoglycoside resistance genes and familial relationships of the aminoglycoside-modifying enzymes. Microbiological Reviews 57:138-163.

Shaw KS, Cruz-Cano R, Jiang C, Malayil L, Blythe D, Ryan P, and Sapkota AR. 2016. Presence of animal feeding operations and community socioeconomic factors impact salmonellosis incidence rates: An ecological analysis using data from the Foodborne Diseases Active Surveillance Network (FoodNet), 2004-2010. Environmental Research 150:166-172. https://doi.org/10.1016/j.envres.2016.05.049

Sivapalasingam S, Friedman CR, Cohen L, and Tauxe RV. 2004. Fresh produce: A growing cause of outbreaks of foodborne illness in the United States, 1973 through 1997. Journal of Food Protection 67:2342-2353. 10.4315/0362-028x-67.10.2342

Skyberg JA, Logue CM, and Nolan LK. 2006. Virulence genotyping of Salmonella spp. With multiplex PCR. Avian Diseases 50:77-81.

Srikantiah P, Bodager D, Toth B, Kass-Hout T, Hammond R, Stenzel S, Hoekstra R, Adams J, Van Duyne S, and Mead PS. 2005. Web-based investigation of multistate salmonellosis outbreak. Emerging Infectious Diseases 11:610.

Srikantiah P, Lay JC, Hand S, Crump JA, Campbell J, Van Duyne MS, Bishop R, Middendor R, Currier M, Mead PS, and Molbak K. 2004. Salmonella enterica serotype Javiana infections associated with amphibian contact, Mississippi, 2001. Epidemiology and Infection 132:273-281.

Peer) reviewing PDF | (2020:05:49215:2:0:NEW 30 Sep 2020) 
1082 Strain E, Luo Y, Pettengill J, Rand HA, Davis S, Payne J, Shpuntoff A, Baugher JD, and Wang

1083

1084

1085

1086

1087

1088

1089

1090

1091

1092

1093

1094

1095

1096

1097

1098

1099

1100

1101

1102

1103

1104

1105

1106

1107

1108

1109

1110

1111

1112

1113

1114

1115

1116

1117

1118

1119

1120

1121

1122

1123

1124

1125

1126

1127
Y. Usage: SNP filtering. Available at https://snp-

pipeline.readthedocs.io/en/latest/usage.html\#snp-filtering (accessed 10 November 2019).

Strawn LK, Danyluk MD, Worobo RW, and Wiedmann M. 2014. Distributions of Salmonella subtypes differ between two U.S. Produce-growing regions. Applied and Environmental Microbiology 80:3982-3991. 10.1128/aem.00348-14

Tableau Software. 2019. Tableau desktop public edition. Available at https://public.tableau.com/en-us/s/.

Tang S, Orsi RH, Luo H, Ge C, Zhang G, Baker RC, Stevenson A, and Wiedmann M. 2019. Assessment and comparison of molecular subtyping and characterization methods for Salmonella. Frontiers in Microbiology 10. 10.3389/fmicb.2019.01591

Timme RE, Pettengill JB, Allard MW, Strain E, Barrangou R, Wehnes C, Van Kessel JS, Karns JS, Musser SM, and Brown EW. 2013. Phylogenetic diversity of the enteric pathogen Salmonella enterica subsp. enterica inferred from genome-wide reference-free SNP characters. Genome Biology and Evolution 5:2109-2123. 10.1093/gbe/evt159

Toth B, Bodager D, Hammond R, Stenzel S, Adams J, Kass-Hout T, Hoekstra R, Mead P, and Srikantiah P. 2002. Outbreak of Salmonella serotype Javiana infections-Orlando, Florida, June 2002. MMWR Morbidity and Mortality Weekly Report 51:683-684.

Tsai CN, and Coombes BK. 2019. The role of the host in driving phenotypic heterogeneity in Salmonella. Trends in Microbiology 27:508-523. 10.1016/j.tim.2019.01.004

U.S. Census Bureau. American factfinder. Available at http://factfinder.census.gov (accessed 13 January 2020).

Van Melderen L. 2001. Molecular interactions of the CcdB poison with its bacterial target, the DNA gyrase. International Journal of Medical Microbiology 291:537-544. https://doi.org/10.1078/1438-4221-00164

Venkat H, Matthews J, Lumadao P, Caballero B, Collins J, Fowle N, Kellis M, Tewell M, White S, Hassan R, Classon A, Joung Y, Komatsu K, Weiss J, Zusy S, and Sunenshine R. 2018. Salmonella enterica serotype Javiana infections linked to a seafood restaurant in Maricopa county, Arizona, 2016. Journal of Food Protection 81:1283-1292. 10.4315/0362-028x.Jfp-17-494

Vosik D, Tewari D, Dettinger L, M'Ikanatha NM, and Shariat NW. 2018. CRISPR typing and antibiotic resistance correlates with polyphyletic distribution in human isolates of Salmonella Kentucky. Foodborne Pathogens and Disease 15:101-108. 10.1089/fpd.2017.2298

Walsh KA, Bennett SD, Mahovic M, and Gould LH. 2014. Outbreaks associated with cantaloupe, watermelon, and honeydew in the United States, 1973-2011. Foodborne Pathogens and Disease 11:945-952. 10.1089/fpd.2014.1812

Wick RR, Judd LM, Gorrie CL, and Holt KE. 2017. Unicycler: Resolving bacterial genome assemblies from short and long sequencing reads. PLOS Computational Biology 13:e1005595. 10.1371/journal.pcbi.1005595

Wick RR, Schultz MB, Zobel J, and Holt KE. 2015. Bandage: Interactive visualization of de novo genome assemblies. Bioinformatics 31:3350-3352. 10.1093/bioinformatics/btv383

Woodward DL, Khakhria R, and Johnson WM. 1997. Human salmonellosis associated with exotic pets. Journal of Clinical Microbiology 35:2786-2790.

Yoshida CE, Kruczkiewicz P, Laing CR, Lingohr EJ, Gannon VPJ, Nash JHE, and Taboada EN. 2016. The Salmonella in silico typing resource (SISTR): An open web-accessible tool for

Peer) reviewing PDF | (2020:05:49215:2:0:NEW 30 Sep 2020) 
1128

1129

1130

1131

1132

1133

1134

1135

1136

1137

1138

1139

1140

1141

1142

1143

1144

1145

1146

1147

1148

1149

1150

1151

1152

1153

1154

1155

1156

1157

1158

1159

1160

1161

1162

1163 rapidly typing and subtyping draft Salmonella genome assemblies. PLOS ONE 11:e0147101. 10.1371/journal.pone.0147101

Zankari E, Allesøe R, Joensen KG, Cavaco LM, Lund O, and Aarestrup FM. 2017. PointFinder: A novel web tool for WGS-based detection of antimicrobial resistance associated with chromosomal point mutations in bacterial pathogens. Journal of Antimicrobial Chemotherapy 72:2764-2768. 10.1093/jac/dkx217

Zankari E, Hasman H, Cosentino S, Vestergaard M, Rasmussen S, Lund O, Aarestrup FM, and Larsen MV. 2012. Identification of acquired antimicrobial resistance genes. Journal of Antimicrobial Chemotherapy 67:2640-2644. 10.1093/jac/dks261

Zhang HZ, Lory S, and Donnenberg MS. 1994. A plasmid-encoded prepilin peptidase gene from enteropathogenic Escherichia coli. Journal of Bacteriology 176:6885. 10.1128/jb.176.22.6885-6891.1994

Zhang S, den Bakker HC, Li S, Chen J, Dinsmore BA, Lane C, Lauer AC, Fields PI, and Deng X. 2019a. SeqSero2: Rapid and improved Salmonella serotype determination using whole-genome sequencing data. Applied and Environmental Microbiology 85:e0174601719. 10.1128/AEM.01746-19

Zhang S, Yin Y, Jones MB, Zhang Z, Deatherage Kaiser BL, Dinsmore BA, Fitzgerald C, Fields PI, and Deng X. 2015. Salmonella serotype determination utilizing high-throughput genome sequencing data. Journal of Clinical Microbiology. 10.1128/jcm.00323-15

Zhang X, Payne M, and Lan R. 2019b. In silico identification of serovar-specific genes for Salmonella serotyping. Frontiers in Microbiology 10. 10.3389/fmicb.2019.00835

Zhou D, Hardt W-D, and Galán JE. 1999. Salmonella Typhimurium encodes a putative iron transport system within the centisome 63 pathogenicity island. Infection and Immunity 67:1974-1981. 10.1128/iai.67.4.1974-1981.1999

Zhou Y, Liang Y, Lynch KH, Dennis JJ, and Wishart DS. 2011. PHAST: A fast phage search tool. Nucleic Acids Research 39:W347-352. 10.1093/nar/gkr485

Zhou Z, Alikhan N-F, Mohamed K, Fan Y, Group AS, and Achtman M. 2019. The EnteroBase user's guide, with case studies on Salmonella transmissions, Yersinia pestis phylogeny and Escherichia core genomic diversity. Genome Research. 10.1101/gr.251678.119

Zhou Z, Alikhan N-F, Sergeant MJ, Luhmann N, Vaz C, Francisco AP, Carriço JA, and Achtman M. 2018. GrapeTree: Visualization of core genomic relationships among 100,000 bacterial pathogens. Genome Research 28:1395-1404. 10.1101/gr.232397.117

Zweifel C, and Stephan R. 2012. Spices and herbs as source of Salmonella-related foodborne diseases. Food Research International 45:765-769. https://doi.org/10.1016/j.foodres.2011.02.024 


\section{Figure 1}

Unrooted neighbor-joining KSNP tree of Tennessee clinical Salmonella ser. Javiana isolates.

Tree was constructed based on core SNPs determined by KSNP3 (Gardner et al., 2015). The optimal tree with the sum of branch length of 5,916.1 is shown. TN clades I (highlighted in purple), II (green), and III (blue) are indicated. The percentage of replicate trees in which the associated taxa clustered together in the bootstrap test are indicated below branches. The tree is drawn to scale, with branch lengths (above branches) representing the number of base differences at core SNP positions per isolate (SNP distance). The analysis involved 112 isolates and 5,870 total SNP positions. 
Tree scale: 1000

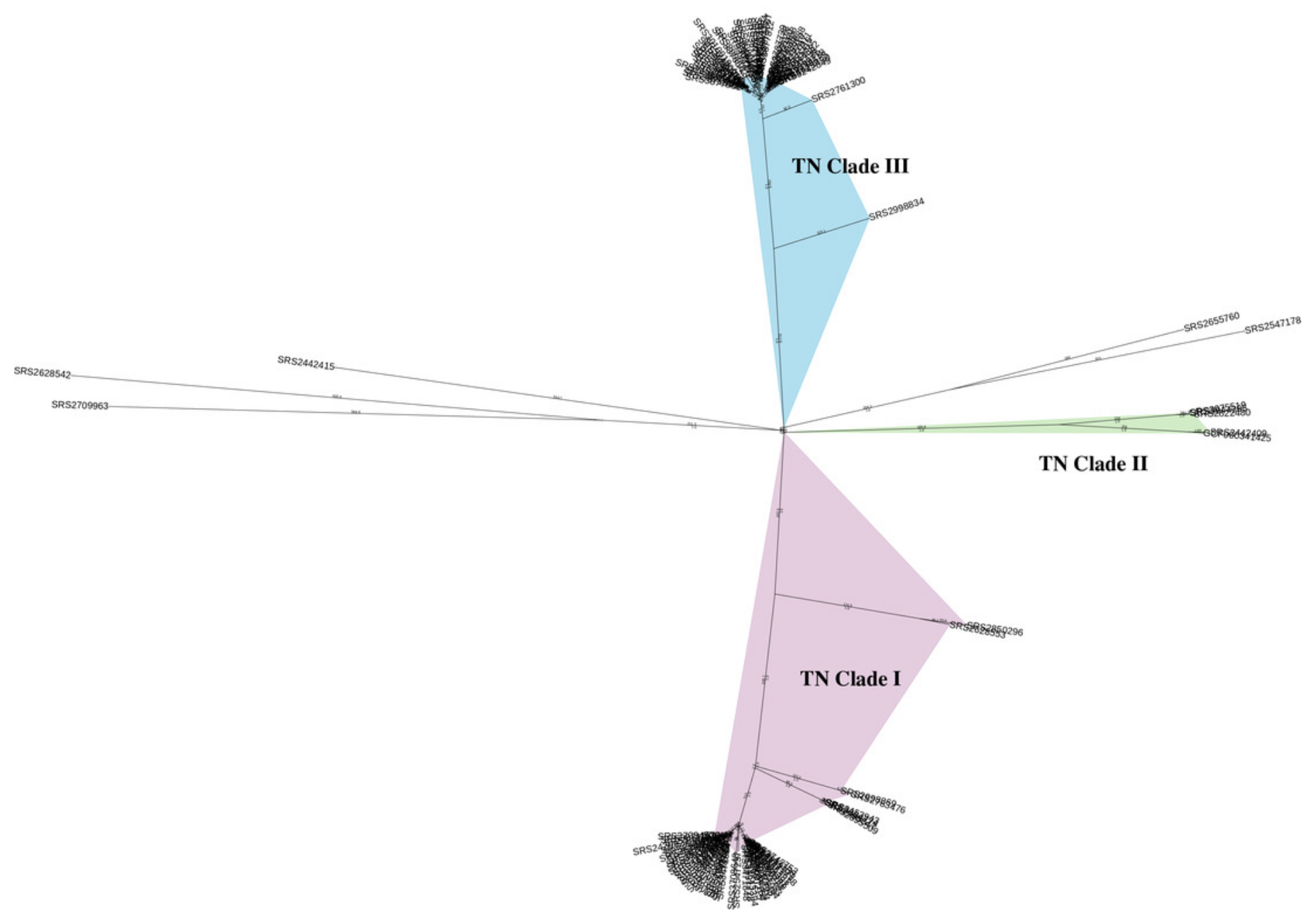




\section{Figure 2}

Neighbor-joining hqSNP trees (Tennessee clades I, II, and III)

Trees were constructed based on hqSNPs identified by the CFSAN SNP Pipeline (Davis et al., 2015). The optimal trees are shown. The percentage of replicate trees in which the associated taxa clustered together in the bootstrap tests that are $\leq 0.8$ are represented by branch color (maximum as green, midpoint as yellow, and minimum as red). The tree is drawn to scale, with branch lengths (above the branches) representing the number of base differences at hqSNP positions per isolate (hqSNP distance). (A) TN clade I. The sum of branch length was 1265.0. The analysis involved 54 isolates and 1261 total hqSNP positions. (B) TN clade II. The sum of branch length was 412.5 . The analysis involved 4 isolates and 426 total hqSNP positions. (C) TN clade III. The sum of branch length was 794.0. The analysis involved 48 isolates and 794 total hqSNP positions. 
A

Tree scale: 10

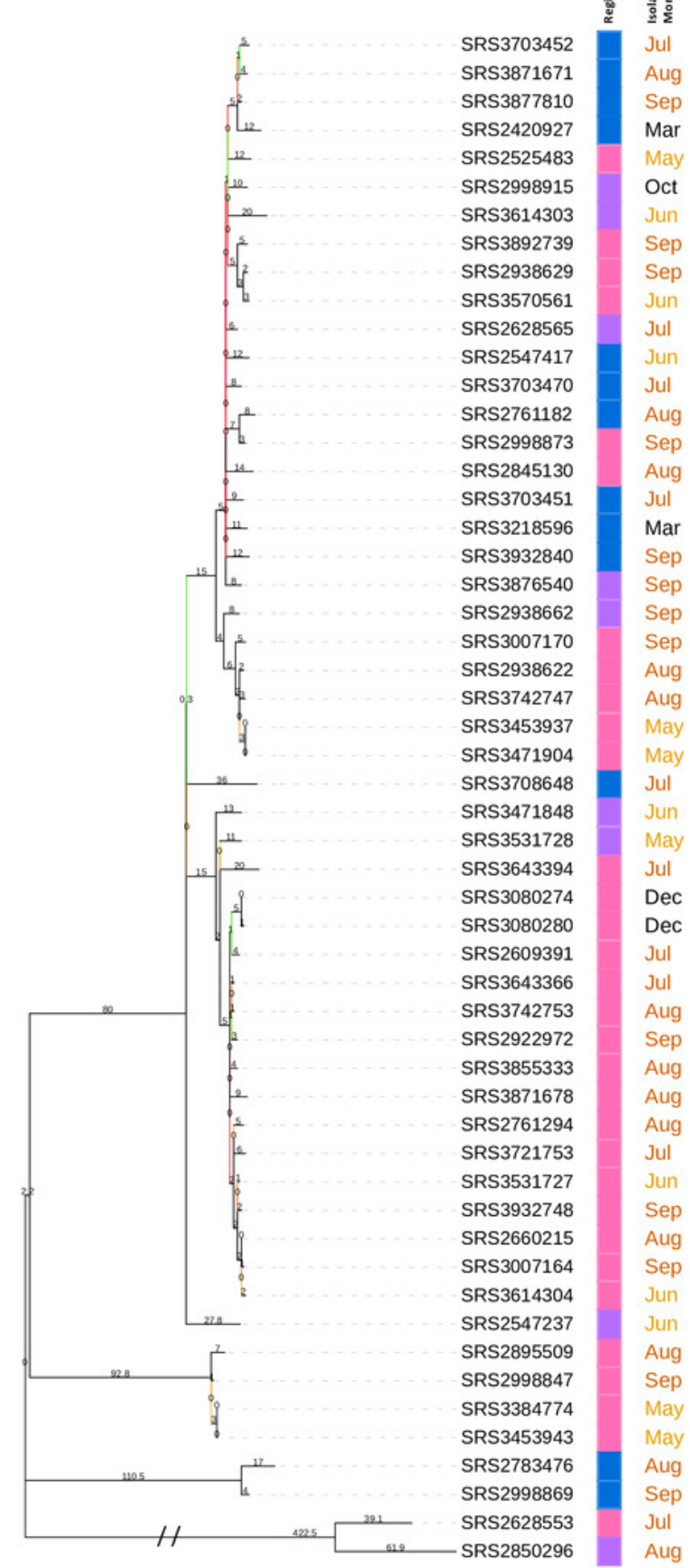

B Tree scale: $10 \longmapsto$

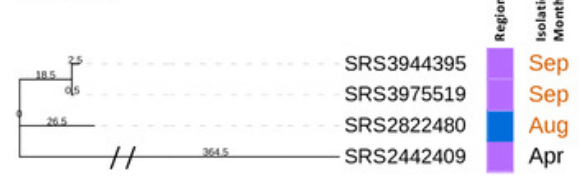

C

Tree scale: $10 \longmapsto$
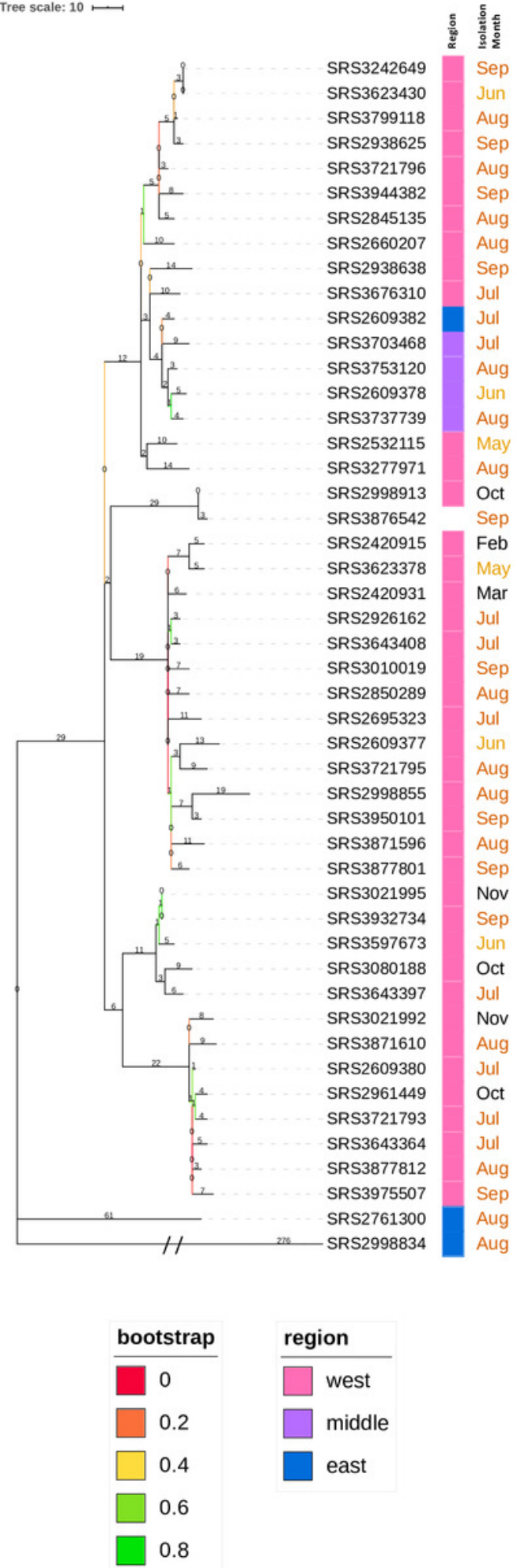


\section{Figure 3}

Circular neighbor-joining KSNP tree of global clinical Salmonella ser. Javiana strains

Tree was constructed based on core SNPs determined by KSNP3 (Gardner et al., 2015). The optimal tree with the sum of branch length of $31,777.6$ is shown. The percentage of replicate trees in which the associated taxa clustered together in the bootstrap test that are $\leq 0.8$ are represented by branch color (maximum as green, midpoint as yellow, and minimum as red). The tree is drawn to scale, with branch lengths (above branches) representing the number of base differences at core SNP positions per isolate (SNP distance). The analysis involved 161 isolates and 30,657 total SNP positions. The three major clades are labeled. HC900 (ceBG) clusters are indicated (590 is not shaded and 204 is shaded in gray). TN isolates belonging to TN clades I, II, and III from our original analysis (Fig. 1) are highlighted in purple, green, and blue, respectively. A standard tree with additional metadata can be found in the supplemental files (Figure S1). 


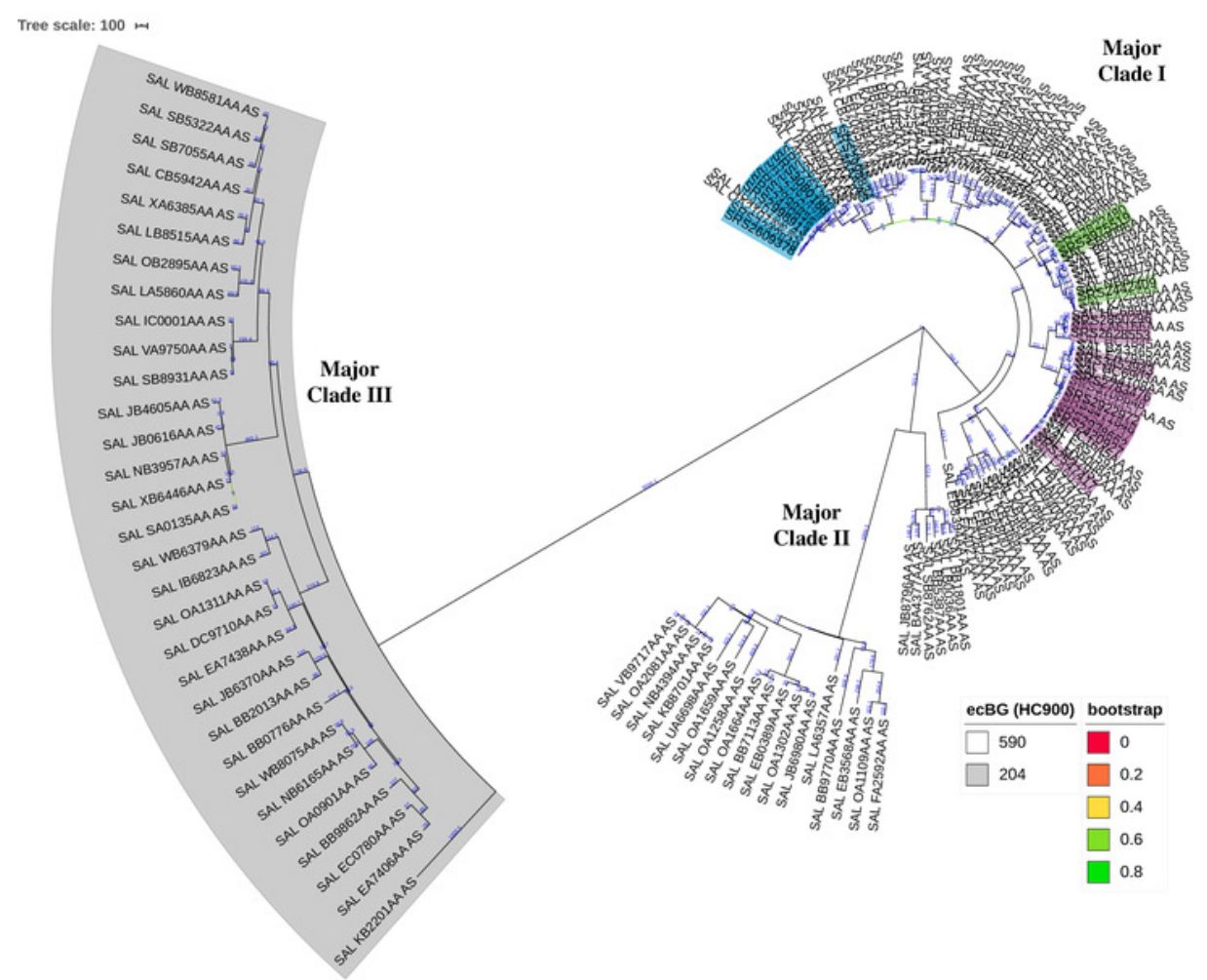




\section{Figure 4}

Minimal spanning trees of Salmonella ser. Javiana and polyphyletic serovar strains

Minimal spanning trees were constructed from cgMLST data using GrapeTree on Enterobase with the improved minimal spanning tree algorithm (MSTree V2). Separate trees were constructed for each (A) Salmonella ser. Javiana, (B) ser. Mississippi, (C) ser. Montevideo, and (D) ser. Newport. Nodes are color coded by ceBG designations (see legend in each panel) and ceBG designations associated with each serovar are in red boxes. Branch lengths (representing cgMLST allelic differences) are shown in red above branches between ceBG clusters. Trees for other serovars are in Figure S2. 

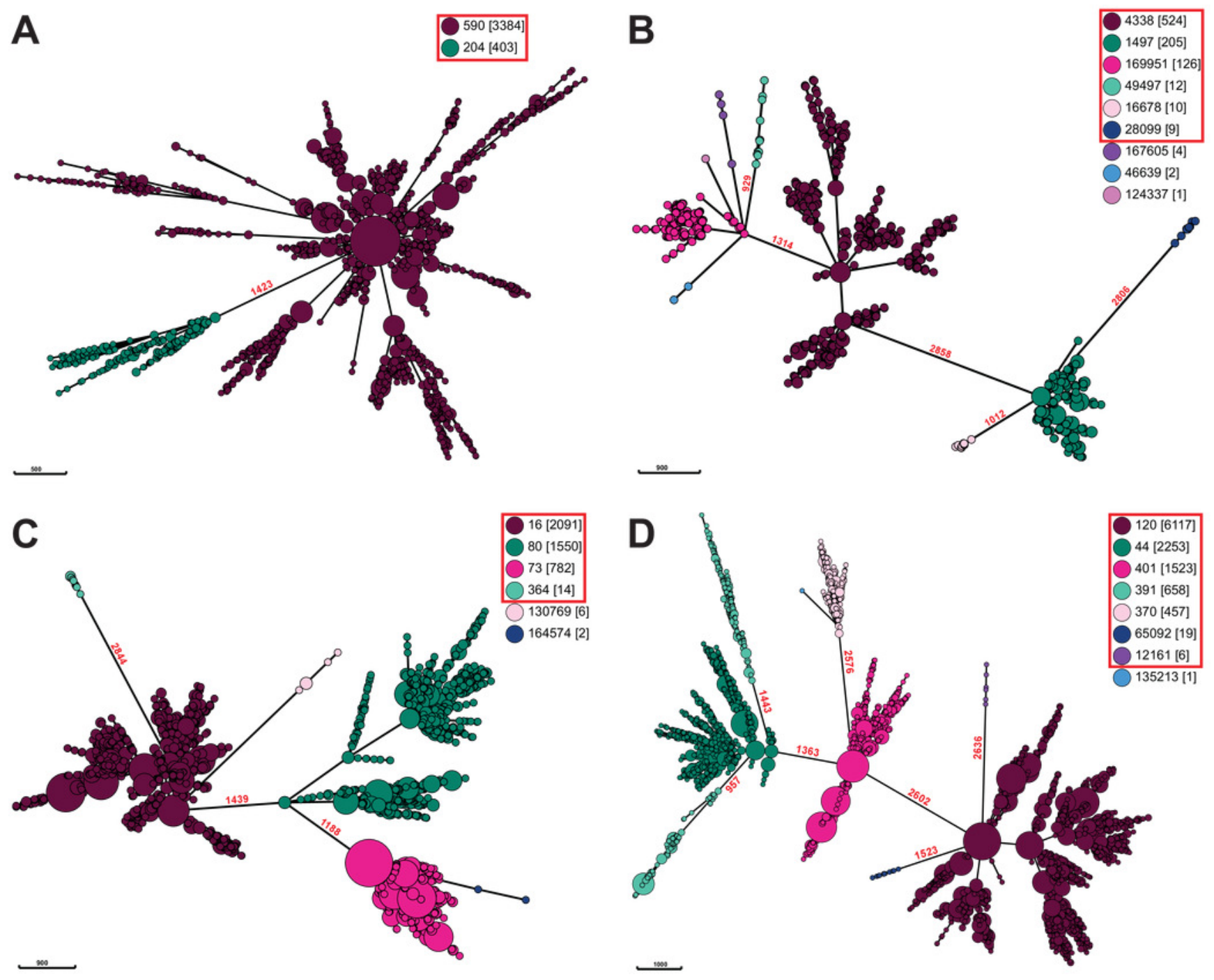


\section{Table $\mathbf{1}$ (on next page)}

Tennessee clade statistics and details

Tennessee clade statistics and details for all isolates and individual clades, including number of isolates, hqSNP analyses details and statistics (reference genome used, mean hqSNP distance and range, and mean percent reads mapped and range), core and accessory genomes as determined by Roary (Page et al., 2015), and loci associated with inclusion in each clade as determined by Scoary (Brynildsrud et al., 2016). 
1 Table 1. Tennessee Clade Statistics and Details

2 Tennessee clade statistics and details for all isolates and individual clades, including number of isolates, hqSNP analyses details and statistics (reference genome used, mean hqSNP distance and range, and mean percent reads mapped and range), core and accessory genomes as determined by Roary (Page et al., 2015), and loci associated with inclusion in each clade as determined by Scoary

5 (Brynildsrud et al., 2016).

\begin{tabular}{|c|c|c|c|c|c|c|c|c|c|}
\hline \multirow{3}{*}{ Clade } & \multirow{3}{*}{$\begin{array}{c}\text { No. } \\
\text { Isolates }\end{array}$} & \multicolumn{4}{|c|}{ hqSNP Analysis } & \multirow{3}{*}{$\begin{array}{c}\text { Core } \\
\text { Genome } \\
\text { (genes) }\end{array}$} & \multirow{3}{*}{$\begin{array}{c}\text { Accessory } \\
\text { Genome } \\
\text { (genes) }\end{array}$} & \multirow{3}{*}{$\begin{array}{c}\text { No. of Locia } \\
\text { Significantly } \\
\text { Associated } \\
\text { with Inclusion } \\
\text { [No. genes] }\end{array}$} & \multirow{3}{*}{$\begin{array}{c}\text { No. of } \\
\text { Significant } \\
\text { Positively } \\
\text { Associated } \\
\text { Loci }^{\mathrm{a}} \\
\text { [No. genes] }\end{array}$} \\
\hline & & & Avg hq & $\begin{array}{l}\text { P distance } \\
\text { ge] }\end{array}$ & & & & & \\
\hline & & Reference & $\begin{array}{l}\text { analyzed } \\
\text { together }\end{array}$ & $\begin{array}{c}\text { analyzed } \\
\text { separately }\end{array}$ & $\begin{array}{c}\text { Mapped } \\
(\%) \text { [range] }\end{array}$ & & & & \\
\hline \multirow{4}{*}{$\begin{array}{c}\text { All } \\
\text { Isolates }\end{array}$} & \multirow{4}{*}{111} & GCF_000341425.1 & $\begin{array}{c}645.3 \\
{[0-1427]}\end{array}$ & - & $\begin{array}{c}96.14 \\
{[90.44-} \\
98.44]\end{array}$ & \multirow{4}{*}{4,022} & \multirow{4}{*}{3,920} & \multirow{4}{*}{-} & \multirow{4}{*}{-} \\
\hline & & SRS2420927 & $\begin{array}{c}627.5 \\
{[0-1416]}\end{array}$ & - & $\begin{array}{c}96.91 \\
{[90.35-} \\
99.70]\end{array}$ & & & & \\
\hline & & SRS2822480 & $\begin{array}{c}631.6 \\
{[0-1404]}\end{array}$ & - & $\begin{array}{c}95.92 \\
{[90.35-} \\
99.77]\end{array}$ & & & & \\
\hline & & SRS3010019 & $\begin{array}{c}630.2 \\
{[0-1429}\end{array}$ & - & $\begin{array}{c}97.59 \\
{[89.55-} \\
99.85]\end{array}$ & & & & \\
\hline \multirow{2}{*}{$\mathrm{I}$} & \multirow{2}{*}{54} & GCF_000341425.1 & $\begin{array}{c}118.8 \\
{[0-605]}\end{array}$ & $\begin{array}{c}119.8 \\
{[0-618]}\end{array}$ & $\begin{array}{c}96.33 \\
{[90.44-} \\
98.38]\end{array}$ & \multirow{2}{*}{4,106} & \multirow{2}{*}{2,513} & \multirow{2}{*}{$\begin{array}{c}153 \\
{[338]}\end{array}$} & \multirow{2}{*}{$\begin{array}{c}54 \\
{[94]}\end{array}$} \\
\hline & & SRS2420927 & $\begin{array}{c}114 \\
{[0-594]}\end{array}$ & $\begin{array}{c}119.4 \\
{[0-631]}\end{array}$ & $\begin{array}{c}97.72 \\
{[90.35-} \\
99.70]\end{array}$ & & & & \\
\hline II & 4 & GCF_000341425.1 & $\begin{array}{c}193.8 \\
{[3-362]}\end{array}$ & $\begin{array}{c}210.3 \\
{[3-396]}\end{array}$ & $\begin{array}{c}97.60 \\
{[96.73-} \\
98.44]\end{array}$ & 4,290 & 322 & $\begin{array}{c}22 \\
{[221]}\end{array}$ & $\begin{array}{c}16 \\
{[207]}\end{array}$ \\
\hline
\end{tabular}




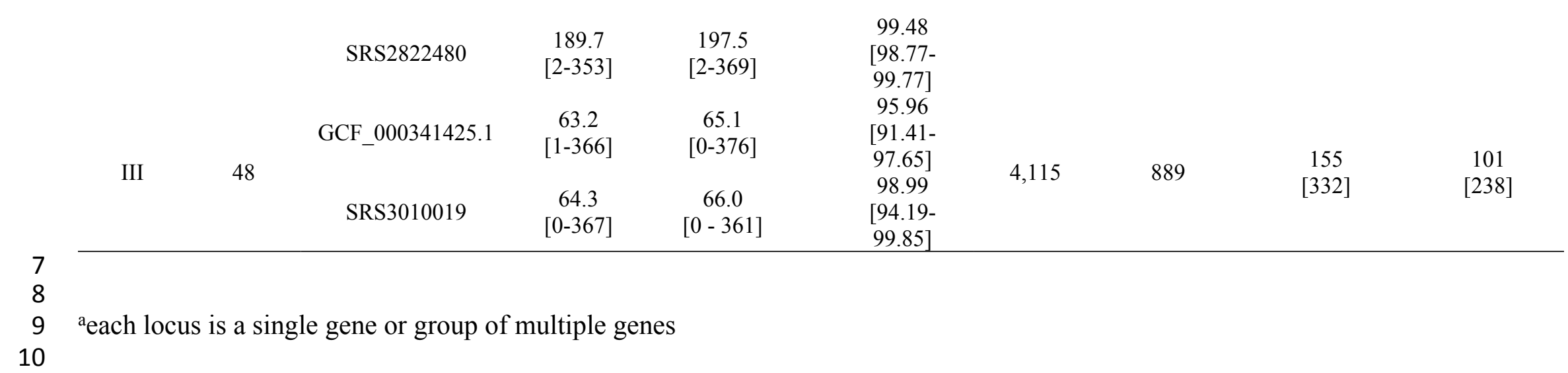




\section{Table 2 (on next page)}

\section{Epidemiological data}

Epidemiological data for all Tennessee isolates and individual TN clades. Percentages are in brackets. 
1 Table 2. Epidemiological Data.

2 Epidemiological data for all isolates and individual clades. Percentages are in brackets.

3

\begin{tabular}{|c|c|c|c|c|c|c|c|c|c|c|c|c|c|c|}
\hline \multirow{2}{*}{ Clade } & \multirow{2}{*}{$\begin{array}{l}\text { No. of } \\
\text { Isolates }\end{array}$} & \multicolumn{3}{|c|}{ Age } & \multicolumn{2}{|c|}{ Sex } & \multicolumn{3}{|c|}{ Isolation Source } & \multicolumn{3}{|c|}{ Geographic Region } & \multicolumn{2}{|c|}{ Isolation Month } \\
\hline & & $<5$ & $5-54$ & $\geq 55$ & Male & Female & Stool & Urine & Blood & West & Middle & East & Jul.-Sep. & Other \\
\hline $\begin{array}{l}\text { All } \\
\text { Isolates }\end{array}$ & 111 & $\begin{array}{c}27 \\
{[24.3]}\end{array}$ & $\begin{array}{c}42 \\
{[37.8]}\end{array}$ & $\begin{array}{c}42 \\
{[37.8]}\end{array}$ & $\begin{array}{c}57 \\
{[51.4]}\end{array}$ & $\begin{array}{c}52 \\
{[46.8]}\end{array}$ & $\begin{array}{c}94 \\
{[84.7]}\end{array}$ & $\begin{array}{c}7 \\
{[6.3]}\end{array}$ & $\begin{array}{c}6 \\
{[5.4]}\end{array}$ & $\begin{array}{c}73 \\
{[65.8]}\end{array}$ & $\begin{array}{c}18 \\
{[16.2]}\end{array}$ & $\begin{array}{c}19 \\
{[17.1]}\end{array}$ & $\begin{array}{c}76 \\
{[68.5]}\end{array}$ & $\begin{array}{c}35 \\
{[31.5]}\end{array}$ \\
\hline I & 54 & $\begin{array}{c}15 \\
{[27.8]}\end{array}$ & $\begin{array}{c}23 \\
{[42.6]}\end{array}$ & $\begin{array}{c}16 \\
{[29.6]}\end{array}$ & $\begin{array}{c}31 \\
{[57.4]}\end{array}$ & $\begin{array}{c}22 \\
{[40.7]}\end{array}$ & $\begin{array}{c}50 \\
{[92.6]}\end{array}$ & $\begin{array}{c}0 \\
{[0.0]}\end{array}$ & $\begin{array}{c}3 \\
{[5.6]}\end{array}$ & $\begin{array}{c}32 \\
{[59.3]}\end{array}$ & $\begin{array}{c}8 \\
{[14.8]}\end{array}$ & $\begin{array}{c}14 \\
{[25.9]}\end{array}$ & $\begin{array}{c}36 \\
{[66.7]}\end{array}$ & $\begin{array}{c}18 \\
{[33.3]}\end{array}$ \\
\hline II & 4 & $\begin{array}{c}0 \\
{[0.0]}\end{array}$ & $\begin{array}{c}2 \\
{[50.0]}\end{array}$ & $\begin{array}{c}2 \\
{[50.0]}\end{array}$ & $\begin{array}{c}3 \\
{[75.0]}\end{array}$ & $\begin{array}{c}1 \\
{[25.0]}\end{array}$ & $\begin{array}{c}4 \\
{[100.0]}\end{array}$ & $\begin{array}{c}0 \\
{[0.0]}\end{array}$ & $\begin{array}{c}0 \\
{[0.0]}\end{array}$ & $\begin{array}{c}0 \\
{[0.0]}\end{array}$ & $\begin{array}{c}3 \\
{[75.0]}\end{array}$ & $\begin{array}{c}1 \\
{[25.0]}\end{array}$ & $\begin{array}{c}3 \\
{[75.0]}\end{array}$ & $\begin{array}{c}1 \\
{[25.0]}\end{array}$ \\
\hline III & 48 & $\begin{array}{c}12 \\
{[25.0]}\end{array}$ & $\begin{array}{c}15 \\
{[31.3]}\end{array}$ & $\begin{array}{c}21 \\
{[43.8]}\end{array}$ & $\begin{array}{c}22 \\
{[45.8]}\end{array}$ & $\begin{array}{c}25 \\
{[52.1]}\end{array}$ & $\begin{array}{c}36 \\
{[75.0]}\end{array}$ & $\begin{array}{c}6 \\
{[12.5]}\end{array}$ & $\begin{array}{c}3 \\
{[6.3]}\end{array}$ & $\begin{array}{c}40 \\
{[83.3]}\end{array}$ & $\begin{array}{c}4 \\
{[8.3]}\end{array}$ & $\begin{array}{c}3 \\
{[6.3]}\end{array}$ & $\begin{array}{c}35 \\
{[72.9]}\end{array}$ & $\begin{array}{c}13 \\
{[27.1]}\end{array}$ \\
\hline
\end{tabular}

4 
Table 3 (on next page)

Putative plasmids

Putative plasmids identified in TN isolates. 
1 Table 3. Putative Plasmids

2 Putative plasmids identified in isolates.

3

\begin{tabular}{|c|c|c|c|}
\hline Plasmid & Isolate(s) (Clade) & Replicon Type & Size (kb) \\
\hline $\mathrm{B}$ & $\begin{array}{c}\text { SRS2628553 (I) } \\
\text { SRS2961449 (III) } \\
\text { SRS2998873 (I) } \\
\text { SRS3570561 (I) } \\
\text { SRS3892739 (I) }\end{array}$ & & 34.8 \\
\hline $\mathrm{C}$ & $\begin{array}{c}\text { SRS2525483 (I) } \\
\text { SRS2547237 (I) } \\
\text { SRS2655760 } \\
\text { SRS3708648 (I) }\end{array}$ & IncFII (p96A) & 67.9 \\
\hline $\mathrm{E}$ & $\begin{array}{l}\text { SRS2442409 (II) } \\
\text { SRS2822480 (II) } \\
\text { SRS3944395 (II) } \\
\text { SRS3975519 (II) }\end{array}$ & IncFII (p14) & $87.4\left(47.5^{*}\right)$ \\
\hline $\mathrm{F}$ & $\begin{array}{l}\text { SRS2783476 (I) } \\
\text { SRS3932840 (I) }\end{array}$ & IncI1 & 97.6 \\
\hline I & SRS2420927 (I) & IncI1 & 92.7 \\
\hline $\mathrm{J}$ & SRS3643397 (III) & IncFII & 68.6 \\
\hline $\mathrm{K}$ & SRS2442415 & IncFIB (pB171) & 94.9 \\
\hline $\mathrm{L}$ & SRS2660207 (III) & IncX4 & 92.4 \\
\hline $\mathrm{M}$ & SRS3453943 (I) & IncN3 & 54.9 \\
\hline $\mathrm{N}$ & SRS2998915 (I) & IncI1 & 24.5 \\
\hline $\mathrm{P}$ & SRS3471904 (I) & & 30.1 \\
\hline $\mathrm{O}$ & SRS2998873 (I) & & 37.7 \\
\hline A & $\begin{array}{l}\text { SRS2998869 (I) } \\
\text { SRS3721796 (III) }\end{array}$ & & 23 \\
\hline $\mathrm{D}$ & $\begin{array}{l}\text { SRS2628553 (I) } \\
\text { SRS2850296 (I) }\end{array}$ & & 86.3 \\
\hline G1 & SRS2547178 & $\operatorname{IncFIB}(\mathrm{S})$ & \\
\hline $\mathrm{Z}$ & SRS2761182 (I) & IncFIB (pB171) & 108 \\
\hline $\mathrm{G} 2$ & SRS3218596 (I) & & 46.3 \\
\hline Q & SRS3742753 (I) & & 64 \\
\hline $\mathrm{Y}$ & SRS2895509 (I) & & 23.5 \\
\hline
\end{tabular}

4 


\section{Table 4(on next page)}

Summary of Scoary results

Summary of genome regions positively associated with inclusion in TN clades I, II, and III, as determined by Scoary (Brynildsrud et al., 2016). Loci that are adjacent or located in close proximity were combined into a single region. Full results are in Data S7. 


\begin{tabular}{|c|c|c|c|c|c|c|c|c|c|c|c|c|c|c|}
\hline \multicolumn{5}{|c|}{ CLADE I } & \multicolumn{5}{|c|}{ CLADE II } & \multicolumn{5}{|c|}{ CLADE III } \\
\hline Ref $^{a}$ & Contig & Start & Stop & MGE $^{\mathbf{b}}$ & Ref & Contig & Start & Stop & MGE & Ref & Contig & Start & Stop & MGE \\
\hline $\mathrm{a}$ & 1 & 105,099 & 105,518 & & $\mathrm{c}$ & 1 & 4,957 & 5,964 & & $\mathrm{~d}$ & 1 & 39 & 620 & \\
\hline $\mathrm{a}$ & 1 & 420,332 & 421,603 & PP & $\mathrm{c}$ & 1 & 385,692 & 385,856 & PP & d & 1 & 25,795 & 25,941 & \\
\hline $\mathrm{a}$ & 1 & 699,132 & 699,236 & & $\mathrm{c}$ & 2 & 47,784 & 63,649 & MGE & d & 1 & 106,264 & 106,698 & \\
\hline $\mathrm{a}$ & 2 & 50,693 & 83,627 & PP & $\mathrm{c}$ & 2 & 74,110 & 92,004 & PP & d & 1 & 246,605 & 246,709 & \\
\hline $\mathrm{a}$ & 2 & 98,106 & 98,423 & & $\mathrm{c}$ & 2 & 130,029 & 149,379 & PP & $d$ & 1 & 639,123 & 639,776 & \\
\hline $\mathrm{a}$ & 2 & 235,502 & 235,738 & & $\mathrm{c}$ & 2 & 191,666 & 192,127 & & d & 1 & 727,239 & 767,712 & MGE \\
\hline $\mathrm{a}$ & 2 & 318,691 & 319,161 & & $\mathrm{c}$ & 2 & 791,655 & 829,140 & PP & $d$ & 1 & $1,082,149$ & $1,118,071$ & PP \\
\hline $\mathrm{a}$ & 3 & 208,678 & 208,803 & & $\mathrm{c}$ & 3 & 150,966 & 168,230 & MGE & d & 1 & $1,225,448$ & $1,226,185$ & \\
\hline $\mathrm{a}$ & 3 & 458,030 & 458,491 & & $\mathrm{c}$ & 4 & 73,904 & 91,513 & MGE & $d$ & 2 & 50,754 & 77,584 & PP \\
\hline $\mathrm{a}$ & 3 & 657,518 & 689,333 & PP & $\mathrm{c}$ & 5 & 66,512 & 66,652 & & d & 2 & 77,581 & 100,603 & MGE \\
\hline $\mathrm{a}$ & 4 & 294,839 & 304,113 & PP & $\mathrm{c}$ & 6 & 134 & 2,179 & & d & 2 & 560,052 & 571,035 & MGE \\
\hline $\mathrm{a}$ & 5 & 195,020 & 195,127 & & $\mathrm{c}$ & 7 & 125,002 & 125,955 & & $\mathrm{~d}$ & 3 & 331,513 & 355,434 & MGE \\
\hline $\mathrm{a}$ & 5 & 235,725 & 236,411 & & $\mathrm{c}$ & 7 & 182,477 & 183,643 & & $d$ & 3 & 492,689 & 492,952 & \\
\hline $\mathrm{a}$ & 5 & 269,009 & 270,619 & & $\mathrm{c}$ & 9 & 79,782 & 80,006 & & d & 4 & 39 & 620 & \\
\hline $\mathrm{a}$ & 5 & 335,429 & 335,854 & & $\mathrm{c}$ & 9 & 99,100 & 99,297 & & d & 4 & 190,030 & 190,365 & \\
\hline $\mathrm{a}$ & 5 & 350,250 & 350,489 & & $\mathrm{c}$ & 13 & 158 & 87,203 & PL & $d$ & 4 & 190,329 & 190,424 & \\
\hline $\mathrm{a}$ & 6 & 60,039 & 60,707 & & $\mathrm{c}$ & 18 & 5,004 & 6,047 & & $d$ & 5 & 1,595 & 7,589 & MGE \\
\hline $\mathrm{a}$ & 6 & 60,750 & 60,974 & & & & & & & $d$ & 5 & 119,382 & 120,038 & \\
\hline $\mathrm{a}$ & 7 & 681 & 1,367 & & & & & & & $d$ & 9 & 684 & 7,579 & $\mathrm{PP}$ \\
\hline $\mathrm{a}$ & 7 & 1,638 & 1,892 & & & & & & & $d$ & 11 & 3,722 & 27,925 & MGE \\
\hline $\mathrm{a}$ & 7 & 197,439 & 205,343 & PP & & & & & & d & 32 & 3 & 236 & \\
\hline $\mathrm{a}$ & 11 & 33,863 & 33,967 & & & & & & & e & 177 & 12 & 149 & MGE \\
\hline $\mathrm{a}$ & 12 & 3,452 & 5,467 & PP & & & & & & $\mathrm{f}$ & 3 & 470,622 & 471,176 & MGE \\
\hline $\mathrm{a}$ & 15 & 1 & 618 & & & & & & & $\mathrm{f}$ & 5 & 231,826 & 232,047 & \\
\hline $\mathrm{a}$ & 15 & 5,734 & 6,108 & & & & & & & $\mathrm{f}$ & 9 & 204,729 & 247,594 & PP \\
\hline $\mathrm{b}$ & 1 & 1,642 & 2,379 & & & & & & & $\mathrm{f}$ & 14 & 75,550 & 76,125 & \\
\hline $\mathrm{b}$ & 1 & 109,453 & 114,795 & PP & & & & & & $\mathrm{g}$ & 1 & 1,076 & 1,198 & MGE \\
\hline $\mathrm{b}$ & 2 & 675,050 & 696,164 & PP & & & & & & $\mathrm{g}$ & 4 & 45 & 617 & \\
\hline $\mathrm{b}$ & 5 & 58,479 & 58,904 & & & & & & & $\mathrm{~g}$ & 31 & 21 & 581 & \\
\hline
\end{tabular}


2 a Reference Isolate: (a) SRS2420927, (b) SRS2628565, (c) SRS2822480, (d) SRS3010019, (e) SRS3643364, (f) SRS3721796, (g) 3 SRS3799118

$4 \quad$ b MGE Region: Prophage (PP), Putative mobile genetic element (MGE), Plasmid (PL) 